\title{
A REAÇÃO “CLICK” NA SÍNTESE DE 1,2,3-TRIAZÓIS: ASPECTOS QUÍMICOS E APLICAÇÕES
}

Luiza Baptista de Oliveira Freitas, Fernando Armini Ruela, Guilherme Rocha Pereira, Rosemeire Brondi Alves e Rossimiriam Pereira de Freitas*

Departamento de Química, Instituto de Ciências Exatas, Universidade Federal de Minas Gerais, CP 702, 31270-901 Belo Horizonte - MG, Brasil

Leandro José dos Santos

Universidade Federal de Viçosa, Campus de Florestal, Rodovia LMG, 818, km 6, s/n, 35690-000 Florestal - MG, Brasil

Recebido em 15/3/11; aceito em 28/6/11; publicado na web em 9/8/11

\begin{abstract}
THE "CLICK” REACTION IN THE SYNTHESIS OF 1,2,3-TRIAZOLES: CHEMICAL ASPECTS AND APPLICATIONS. The Copper-catalyzed azide-alkyne cycloaddition (CuAAC), often referred to as "click" reaction, has become a very popular reaction in the last years. It affords exclusively 1,4-disubstituted 1,2,3-triazoles and has been widely used to connect readily accessible building blocks containing various functional groups. The great success of this reaction is based on the fact that it is general, virtually quantitative and very robuste. The scope of this copper-catalyzed synthesis is extraordinary and the reaction has found numerous applications in many research fields, including biological chemistry and materials science. In this review, the main chemical aspects and applications of the "click" reaction in the synthesis of 1,2,3-triazoles are presented.
\end{abstract}

Keywords: "click" reaction; cycloaddition; triazole.

\section{INTRODUÇÃO}

A química "click" é um conceito introduzido em 2001 por K. Barry Sharpless ${ }^{1}$ para descrever reações termodinamicamente favoráveis que, realizadas em laboratório, são capazes de conectar duas moléculas de forma muito simples e com altos rendimentos, sendo de grande aplicabilidade. Segundo Sharpless, para ser caracterizada como química "click" uma reação deve ser rápida, estereoespecífica (mas não necessariamente enantiosseletiva) e produzir produtos secundários inofensivos, que podem ser removidos preferencialmente sem uso de cromatografia. Além disso, uma reação "click" deve ser realizada sem solventes ou que estes sejam atóxicos e inofensivos, usar materiais de partida estáveis e de simples obtenção e não necessitar, por exemplo, de cuidados especiais (idealmente o processo deve ser insensível a oxigênio e água). Sharpless introduziu esse conceito para defender a necessidade de que químicos sintéticos se voltem para reações simples como estratégia de obtenção de bibliotecas de compostos a serem testados, tanto na área farmacêutica quanto na de materiais. No seu artigo original para introdução desta nova filosofia química, ${ }^{1}$ o autor descreve uma série de reações clássicas em química orgânica que poderiam se enquadrar nesta categoria, com destaque para a abertura nucleofílica de epóxidos e aziridinas, para as adições a ligações múltiplas carbono-carbono, para reações de cicloadição, dentre outras. ${ }^{1}$

Dentre as reações compreendidas nesse universo "click" proposto por Sharpless, o exemplo perfeito e que hoje é comumente tratado como reação "click" é a cicloadição (C) 1,3-dipolar entre um alcino terminal (A) e uma azida orgânica (A) catalisada por cobre $(\mathrm{Cu}(\mathrm{I}))$, com formação regioespecífica de 1,2,3-triazóis-1,4-dissubstituídos, também conhecida como reação CuAAC. O número de publicações envolvendo o uso da reação "click" para a preparação de novas moléculas tem crescido exponencialmente nos últimos anos e uma

*e-mail: rossi@netuno.lcc.ufmg.br revisão exaustiva do assunto não seria aqui possível. Este artigo de revisão tem, portanto, o objetivo de apresentar alguns aspectos químicos atualizados sobre a reação "click" na síntese de 1,2,3-triazóis assim como as principais considerações mecanísticas existentes, as possíveis fontes de cobre e solventes para a reação, a influência da estrutura dos alcinos e azidas na reatividade, bem como possíveis subprodutos que podem ser formados. Além disso, serão mostrados alguns exemplos selecionados de aplicações da reação CuAAC tanto na área biológica quanto na área de materiais, para comprovar que a ideia original de Sharpless era brilhante: a "reinvenção" de reações químicas "simples e antigas" pode propiciar grande avanço em várias áreas de pesquisa, com a descoberta de inúmeras novas moléculas com funções variadas.

\section{A REAÇÃO “CLICK” NA SÍNTESE DE 1,2,3-TRIAZÓIS}

O 1,2,3-triazol é um heterociclo de origem exclusivamente sintética, não ocorrendo na natureza. ${ }^{2}$ Um dos métodos clássicos para sua obtenção é a cicloadição térmica 1,3-dipolar envolvendo azidas orgânicas e alcinos terminais ou internos. Originalmente observada por Michael, ${ }^{3}$ em 1893, e desenvolvida por Huisgen, ${ }^{4}$ em 1967, esta reação concertada apresenta vários problemas como a necessidade de longos tempos reacionais e de altas temperaturas. Além disso, apresenta baixos rendimentos e há formação de uma mistura de regioisômeros triazólicos 1,4 e 1,5-dissubstituídos quando alcinos assimétricos estão envolvidos (Esquema 1).

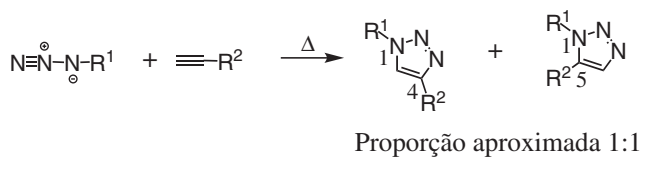

Esquema 1. Regioisômeros obtidos via cicloadição térmica [3+2] clássica de Huisgen 
A aplicabilidade desta reação começou a mudar quando estudos realizados concomitantemente pelos grupos de Meldal $^{5}$ e de Sharpless, ${ }^{6} \mathrm{em} 2002$, mostraram que a utilização de $\mathrm{Cu}(\mathrm{I})$ acelerava a reação de forma surpreendente, com um aumento na taxa de velocidade na ordem de 7 vezes. Além disso, a reação nestas condições levava à formação exclusiva do regioisômero 1,4-dissubstituído. ${ }^{5,6}$ Em relação ao método clássico de cicloadição 1,3-dipolar de Huisgen, a reação na presença de cobre utilizava condições muito mais brandas, resultava em rendimentos muito altos e era de fácil elaboração. Alcinos e azidas são facilmente preparados em laboratório e estão entre os grupos funcionais mais inertes em diversas condições de reação e mesmo em ambientes aquosos. Assim, uma reação envolvendo estes dois grupos funcionais pode ocorrer facilmente mesmo na presença de outros grupos reativos nas moléculas envolvidas. O heterociclo triazólico obtido na reação é muito estável e tem similaridade com a função amida encontrada na natureza. Todas estas condições reunidas fizeram com que a cicloadição de Huisgen "revigorada" pela presença de cobre tenha se tornado uma das reações mais utilizadas atualmente por muitos grupos de pesquisa em estratégias de síntese de 1,2,3-triazóis 1,4-substituídos, tendo se tornado, portanto, o protótipo da química "click" e, como já citado, recebido o nome comum de reação "click" (Esquema 2). ${ }^{7} \mathrm{~A}$ impressionante velocidade com que esta reação tem sido empregada em várias áreas de pesquisa, além da síntese orgânica, é um indicador claro do seu amplo potencial de aplicação.

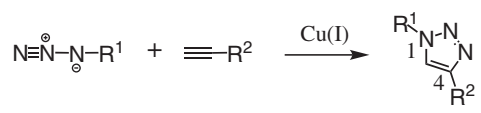

Esquema 2. A reação "click” ou CuAAC

\section{ASPECTOS MECANÍSTICOS DA REAÇÃO “CLICK”}

Como já mencionado, a presença de $\mathrm{Cu}(\mathrm{I})$ muda drasticamente a velocidade da formação do 1,2,3-triazol. Isso se deve à alteração do mecanismo e do caminho da reação. A exata função do cobre nesta reação tem sido objeto de estudo de vários pesquisadores nos últimos 10 anos e várias discussões são encontradas na literatura. ${ }^{8-13}$

A reação térmica de Huisgen para a formação destes heterociclos é altamente exotérmica ( $\Delta H^{o}$ entre -50 e $\left.-65 \mathrm{Kcal} \mathrm{mol}^{-1}\right)$, mas a sua alta energia de ativação (aproximadamente $25 \mathrm{Kcal} \mathrm{mol}^{-1}$ para a reação entre propino e azida de metila) resulta em velocidades de reação muito baixas para reagentes não ativados, mesmo em altas temperaturas. Além disso, desde que as diferenças de energia entre os orbitais de fronteira HOMO e LUMO de azidas e alcinos são de magnitude similares, os mecanismos operando por dipolo-HOMO e dipolo-LUMO ocorrem simultaneamente nestas reações, produzindo misturas regioisoméricas de triazóis. A presença de cobre, no entanto, transforma o mecanismo concertado em uma sequência de rápidas etapas envolvendo intermediários polares cujas estruturas definem a regioespecificidade da reação e cujas energias de formação determinam a velocidade da reação. De forma bastante simplificada (Esquema 3) na presença de $\mathrm{Cu}(\mathrm{I})$ ocorre a formação inicial do acetileto do metal (a), que se complexa também ao nitrogênio nucleofílico da azida (b), favorecendo o ataque do carbono do tipo $\beta$-vinilidênico do acetileto de $\mathrm{Cu}(\mathrm{I})$ ao nitrogênio eletrofílico terminal da azida orgânica, com formação da primeira ligação C-N e obtenção do metalociclo instável (c). Apesar de intrigante, as primeiras propostas mecanísticas envolvendo intermediários de cobre mononucleares defendiam a existência deste metalociclo pouco comum, sendo que Fokin e colaboradores ${ }^{13}$ mostraram, por meio de cálculos teóricos de densidade funcional, que a estrutura otimizada do mesmo possuía um ângulo entre as ligações $\mathrm{Cu}=\mathrm{C}=\mathrm{C}$ de $134,9^{\circ}$. A contração do anel leva à formação do triazolila de cobre (d), que sofre protonólise para formação do 1,2,3-triazol 1,4-dissubstituído (e)..$^{6,12,13}$

Embora este mecanismo simplificado esclareça as etapas elementares envolvendo formação e quebra de ligações, inúmeras considerações teóricas e experimentais obtidas até o momento mostram que a reação é muito mais complexa do que isso e deve envolver intermediários de cobre dinucleares. ${ }^{7,11,12,14,15}$ Uma proposta mais detalhada aceita atualmente está representada pelo ciclo catalítico, Esquema 4. Cálculos teóricos indicam que a complexação $\pi$ entre o $\mathrm{Cu}(\mathrm{I})$ e o alcino terminal diminui o $\mathrm{pKa}$ do hidrogênio para 9,8 unidades, o que possibilita a desprotonação em um sistema aquoso sem a necessidade de se adicionar base,${ }^{13}$ facilitando a formação do acetileto de cobre ( 2 e 3). Existem várias discussões sobre a natureza deste intermediário e evidências indicam que podem estar envolvidos dois centros metálicos, um ou dois alcinos ligantes e outros ligantes mais lábeis que competem com a ligação com a azida. ${ }^{16}$ Como citado anteriormente, após a formação do acetileto de cobre (2 e 3) ocorre a complexação com a azida, gerando o complexo azida-acetileto (4). A proporção 1:1 de azida/alcino no intermediário (4) foi sugerida experimentalmente por Sun e $\mathrm{Wu}^{17}$ por meio da análise da reação por infravermelho, porém estão descritas outras propostas estruturais. Neste intermediário o cobre tem um efeito sinérgico nos sítios reativos, tornando o nitrogênio terminal da azida mais eletrofílico e o carbono do tipo $\beta$-vinilidênico mais nucleofílico, o que favorece o ataque para formação do metalociclo (5). Esta etapa, que é endotérmica e define a regioespecificidade da reação, tem energia de ativação (Ea) calculada de aproximadamente $15 \mathrm{kcal} \mathrm{mol}^{-1}$, que é menor do que a Ea para a reação não catalisada (cerca de $26 \mathrm{kcal} \mathrm{mol}^{-1}$ ). Isto explica o grande aumento da velocidade da reação quando comparado com o processo térmico. ${ }^{11,13}$ Embora o metalociclo (5) tenha sido proposto em vários trabalhos com apenas uma espécie metálica, ${ }^{5,6,13}$ diversos pesquisadores sugerem que a ciclização ocorra com dois centros metálicos. ${ }^{914,16,18}$ Em seguida, ocorre a contração do anel por uma associação transanular do par de elétrons não ligantes do N-1 com o orbital antiligante de C-5 fornecendo o triazolila de cobre (6-7). Este intermediário pode ser isolado quando estão presentes grupos protetores volumosos nos substratos. ${ }^{12} \mathrm{Na}$ última etapa do mecanismo ocorre a protonação do intermediário triazoíla de cobre (6-7) com a formação do produto final $(\mathbf{8})$ e regeneração do catalisador. Esta protonação pode ocorrer por meio da interação com uma base protonada ou com o próprio solvente, porém a presença de outros eletrófilos pode levar à formação de 1,2,3-triazóis-1,4,5-trissubstituídos. ${ }^{16}$

A grande dificuldade em se estabelecer as estruturas exatas dos intermediários envolvidos na reação "click" e a ordem global da mesma advêm da grande tendência das espécies de cobre de formarem complexos polinucleares e da facilidade de trocas de ligantes que este metal apresenta. Aparentemente, múltiplas espécies de organocobre se encontram em rápido equilíbrio umas com as outras no meio reagente. Embora isto dificulte a determinação exata do mecanismo

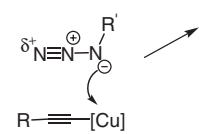

(a)

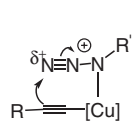

(b)

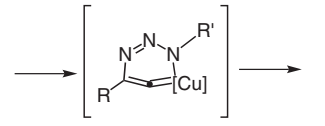

(c)

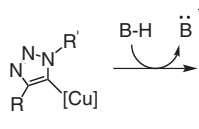

(d)

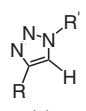

(e) 


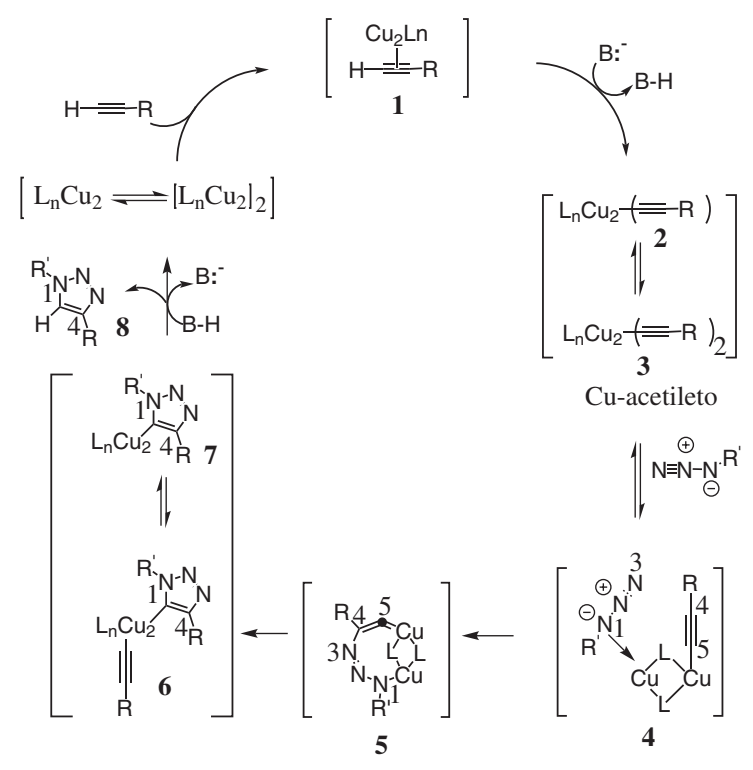

L =ligante; $\mathrm{B}=$ base ou solvente.

Esquema 4. Mecanismo proposto para a reação CuAAC

da reação $\mathrm{CuAAC}$, aparentemente esta dinâmica exclusiva do cobre é responsável pelo fato de que a reação "click" se adapte a tantas condições diferentes de solventes e de que o cobre seja o único metal que promova esta reação de forma tão eficiente. Outros metais conhecidos como catalisadores de reações de alcinos $(\mathrm{Ag}(\mathrm{I}), \mathrm{Pd}(0 /$ II), $\mathrm{Pt}(\mathrm{II}), \mathrm{Au}(\mathrm{I} / \mathrm{III}), \mathrm{Hg}(\mathrm{II})$, entre outros) se mostraram ineficazes para produzir triazóis em rendimentos aceitáveis em síntese. ${ }^{12}$ Em 2005, complexos de rutênio do tipo ciclopentadienila mostraram-se eficientes para catalisar a formação de triazóis 1,5-dissubstituídos a partir de alcinos e azidas, ${ }^{19}$ entretanto, aparentemente esta reação, denominada RuAAC por analogia à reação "click", é mecanisticamente bastante distinta da $\mathrm{CuAAC}$, assim como sua sensibilidade a solventes e a fatores estéricos.

\section{FONTES DE COBRE E CATALISADORES}

Apesar do $\mathrm{Cu}(\mathrm{I})$ ser a espécie envolvida durante o processo de catálise, as fontes de cobre utilizadas para a reação "click" podem variar. O grupo de Meldal originalmente utilizou sais de $\mathrm{Cu}(\mathrm{I})$ na reação, ${ }^{5}$ enquanto o grupo de Sharpless investigou o uso de sais de $\mathrm{Cu}$ (II) na presença de um redutor para formar a espécie catalítica in situ. ${ }^{6}$ Atualmente, a escolha da fonte de cobre é feita com base nas necessidades especiais de cada reação e são inúmeras as combinações já descritas na literatura. ${ }^{11}$

Como fonte direta de íons cuprosos, os sais de $\mathrm{Cu}(\mathrm{I})$ (iodeto, ${ }^{5,20,21}$ brometo, ${ }^{22}$ cloreto $^{23}$ ou acetato ${ }^{24,25}$ ) e complexos de coordenação como $\left[\mathrm{Cu}\left(\mathrm{CH}_{3} \mathrm{CN}\right)_{4}\right] \mathrm{PF}_{6}{ }^{26}$ e $\left[\mathrm{Cu}\left(\mathrm{CH}_{3} \mathrm{CN}\right)_{4}\right] \mathrm{OTf}^{27}$ têm sido usados mais frequentemente. Geralmente, a reação com sais de $\mathrm{Cu}(\mathrm{I})$ demanda o uso de uma base nitrogenada ou de aquecimento para promover a formação inicial do acetileto de cobre, uma vez que, apesar destes sais de cobre serem solúveis nos solventes empregados, eles se encontram geralmente como aglomerados estáveis (por exemplo, $\mathrm{Cu}_{2} \mathrm{I}_{3}$ , $\mathrm{Cu}_{4} \mathrm{I}_{5}-\mathrm{Cu}_{5} \mathrm{I}_{6}^{-}$e $\left.\mathrm{Cu}_{6} \mathrm{I}_{7}^{-}\right){ }^{11,12}$ Desta forma, o $\mathrm{Cu}(\mathrm{I})$ não está inicialmente disponível para promover a catálise da cicloadição. ${ }^{11}$ A sonicação é um outro artifício empregado para promover o rompimento dos agregados. ${ }^{20}$ Outra desvantagem do uso de sais de $\mathrm{Cu}$ (I) é sua instabilidade termodinâmica, o qual pode ser oxidado para $\mathrm{Cu}(\mathrm{II})$ e/ou sofrer desproporcionamento para cobre metálico e $\mathrm{Cu}$ (II) no decorrer da reação. Desta forma, muitos procedimentos requerem atmosfera inerte e condições anidras. O uso de sais de iodo é pouco recomendado devido à habilidade do íon iodeto de atuar como ligante para o metal, promovendo a formação de complexos polinucleares de acetiletos, os quais interferem no ciclo catalítico produtivo. ${ }^{12}$ Além disso, sob certas condições, 5-iodotriazóis já foram isolados. ${ }^{28}$ Altas concentrações de íons cloreto em água também já se mostraram prejudiciais à reação. Dessa forma, o uso de brometo ou acetato cuproso é o mais indicado para reações em meio aquoso, onde se empregam diretamente íons $\mathrm{Cu}(\mathrm{I})$ como fonte de cobre. ${ }^{12}$

Uma alternativa que se tornou muito popular e prática para a reação "click" é o uso de sais estáveis de $\mathrm{Cu}(\mathrm{II})$, como o sulfato penta-hidratado $\left(\mathrm{CuSO}_{4} \cdot 5 \mathrm{H}_{2} \mathrm{O}\right)$ ou o acetato de $\mathrm{Cu}(\mathrm{II})$, na presença de um agente redutor fraco como o ascorbato de sódio. Desenvolvida originalmente por Sharpless e colaboradores, ${ }^{6}$ a metodologia apresenta algumas vantagens importantes: a reação sofre menor interferência do oxigênio atmosférico (qualquer oxigênio dissolvido é rapidamente reduzido), podendo até mesmo ser realizada em frasco aberto, além de não haver necessidade de se utilizar ligantes ou aditivos. Este método foi desenvolvido em meio aquoso, uma vez que o sulfato de cobre e o agente redutor são hidrossolúveis. Há evidências de que a água seja o solvente ideal capaz de preservar o acetileto de cobre em seu estado reativo quando o mesmo é formado in situ..$^{12}$ Apesar de muitas reações com sais de $\mathrm{Cu}(\mathrm{II})$ serem feitas em água, a grande maioria dos relatos utiliza uma extensa variedade de solventes ou misturas de solventes, como será discutido posteriormente. É importante ressaltar que a formação de acetiletos de cobre reativos (e não na forma de aglomerados) na presença da azida orgânica é essencial para o sucesso da reação. A complexação do acetileto com a azida é fundamental para prevenir a formação de acetiletos poliméricos, que contribuem para o insucesso da reação.

Como fonte alternativa de cobre, pode-se ainda citar o uso de cobre metálico (fio, limalha, pó ou aglomerado) utilizado na presença ou ausência de sulfato de cobre ${ }^{29-31}$ que, após redução, leva à formação da espécie reativa $\mathrm{Cu}(\mathrm{I})$. Geralmente, observa-se um período de latência até que a espécie reativa seja formada. A irradiação por micro-ondas pode reduzir significativamente o tempo de latência para este método ${ }^{29} \mathrm{e}$ o tempo de reação para os demais métodos..$^{32,33}$ Cravotto e colaboradores ${ }^{34,35}$ mostraram resultados promissores na associação de micro-ondas e ultrassom para a CuAAC.

Além dessas fontes de cobre citadas, muitos outros complexos de cobre envolvendo diferentes ligantes têm sido relatados. Embora não sejam necessários para o efeito catalítico do $\mathrm{Cu}(\mathrm{I})$, os ligantes são frequentemente empregados para aumentar a velocidade da reação e para proteger o $\mathrm{Cu}(\mathrm{I})$ de oxidação na presença eventual de oxigênio. ${ }^{12}$ Dentre os complexos de coordenação usados, podem ser citados aqueles contendo fosfinas como ligantes, como $\mathrm{CuP}(\mathrm{EtO})_{3} \mathrm{I},{ }^{36} \mathrm{Cu}\left(\mathrm{PPh}_{3}\right)_{3} \mathrm{Br},{ }^{36-38}$ e $\mathrm{Cu}\left(\mathrm{PPh}_{3}\right)_{2} \mathrm{OAc},{ }^{39}$ complexos com carbenos $\mathrm{N}$-heterocíclicos ${ }^{40}$ e complexos contendo aminas terciárias como ligantes. ${ }^{36,41,42}$ Este último grupo é o mais importante já que as aminas terciárias centrais podem funcionar tanto como base quanto como centro doador na coordenação com o metal. A necessidade desses ligantes é particularmente importante no caso de reações $\mathrm{CuAAC}$ envolvendo biomoléculas, que são geralmente realizadas em meio aquoso, em baixas concentrações de reagente e sem aquecimento. Nestas condições, já ficou evidenciado que a velocidade da reação na ausência de um ligante acelerador é muito baixa. O primeiro ligante utilizado em bioconjugação foi o TBTA (tris-benziltriazolilmetilamina) (Figura 1) cuja complexação com o cobre resulta em um aumento da densidade eletrônica do metal e, consequentemente, em uma aceleração do processo de catálise. ${ }^{41} \mathrm{O}$ TBTA não é, entretanto, uma base suficientemente forte para romper os aglomerados dos sais de $\mathrm{Cu}(\mathrm{I})$ e por isso não é utilizado como base na metodologia de Meldal e colaboradores. Outros ligantes aminados mais solúveis em água e o ligante comercialmente disponível bato* 
ou batofenantrolina (4,7-difenil-1,10-fenantrolina) (Figura 1) também apresentam resultados favoráveis para a CuAAC.

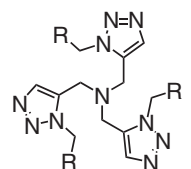

$$
\begin{aligned}
9 \mathrm{R} & =\text { benzila TBTA } \\
10 \mathrm{R} & =\mathrm{CH}_{2} \mathrm{CH}_{2} \mathrm{CH}_{2} \mathrm{OH} \text { THPTA } \\
11 \mathrm{R} & =\text { terc-butila TTTA }
\end{aligned}
$$

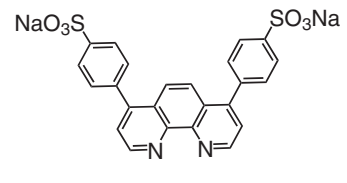

bato

Figura 1. TBTA, derivados e batofenantrolina (bato): ligantes utilizados em $C u A A C$

Apesar do grande número de possibilidades experimentais, não existe uma correlação óbvia entre o tipo de método empregado e o rendimento da reação e, por isso, cada caso deve ser otimizado. Alguns exemplos de diferentes fontes de cobre para a realização da CuAAC estão mostrados na Tabela 1 , mas muitas outras são conhecidas. ${ }^{11}$

\section{PRINCIPAIS SOLVENTES EMPREGADOS}

Nos primeiros estudos envolvendo a reação CuAAC, Sharpless e colaboradores relataram o uso de água como solvente, com ou sem a adição de cossolvente (os solventes clássicos inicialmente utilizados foram terc-butanol e etanol). ${ }^{6} \mathrm{O}$ uso da água, segundo os autores, apresentava como vantagens o fato de se tratar de um solvente benigno, universal, que dissolvia os sais inorgânicos de $\mathrm{Cu}$ (II) e o ascorbato de sódio, estabilizava o acetileto e possuía capacidade de absorver a grande quantidade de calor gerada neste tipo de reação. ${ }^{1}$ Estes pesquisadores enfatizavam ainda que não havia necessidade dos materiais de partida estarem dissolvidos no solvente da reação observando que, na maioria dos casos, a reação acontecia de forma muito eficiente desde que fosse mantida agitação forte. Os resultados desses primeiros estudos mostravam que a reação, mesmo com os materiais de partida apenas suspensos em água, ocorria rapidamente e com excelentes rendimentos. ${ }^{1}$ Como já citado, a reação CuAAC também foi estudada paralelamente por Meldal e seu grupo na mesma época, ${ }^{5}$ os quais também relataram ótimos rendimentos usando solventes orgânicos, uma vez que os mesmos dissolviam de forma mais eficaz os sais de $\mathrm{Cu}(\mathrm{I})$ e as aminas que tinham que ser adicionadas como ligantes/aditivos.
Seguindo essas duas principais vertentes, vários solventes têm sido usados na reação CuAAC. Solventes orgânicos, tais como THF, DMSO, DMF, $\mathrm{CHCl}_{3}$, piridina, tolueno, $\mathrm{CH}_{2} \mathrm{Cl}_{2}$, acetonitrila, entre outros, continuam a serem usados nos casos onde se usa $\mathrm{CuI}$, $\mathrm{Cu}\left(\mathrm{CH}_{3} \mathrm{CN}\right)_{4} \mathrm{PF}_{6}, \mathrm{CuIP}(\mathrm{OEt})_{3}$ ou outros sais de $\mathrm{Cu}(\mathrm{I})$ que apresentam maior solubilidade neste tipo de meio, acompanhados de um excesso de base. ${ }^{11,51}$ Há algumas generalizações que podem ser feitas, por exemplo, solventes como o DMF demonstram grande aplicabilidade em reações "click" destinadas ao estabelecimento de ligações entre blocos de polímeros. ${ }^{52-56}$ Já no caso do método preconizado por Sharpless, o do "ascorbato aquoso", o número de cossolventes já testados é bastante variado e inclui acetona, THF, DMSO, DMF, $\mathrm{MeOH}, \mathrm{AcOEt}$, dentre outros. ${ }^{11} \mathrm{Kim}$ e colaboradores ${ }^{57}$ relataram o sucesso do uso de $\mathrm{CH}_{2} \mathrm{Cl}_{2}$ como cossolvente para reações que deram rendimentos muito baixos ou não se realizaram na presença de outros cossolventes.

Hoje em dia se sabe que a maioria dos solventes conhecidos, puros ou na forma de sistemas bifásicos, podem ser empregados na reação "click" ${ }^{58}$ Apesar desta versatilidade, a decisão definitiva sobre o tipo de solvente a ser usado depende não só da fonte de cobre empregada mas também das propriedades dos reagentes em questão e, algumas vezes, a realização de um estudo prévio com diversos solventes é essencial. ${ }^{21}$ Muitos autores defendem que a solubilidade dos reagentes é vital para o sucesso da reação, contrariamente ao preconizado por Sharpless em seus primeiros estudos envolvendo água. Por exemplo, Fleming e colaboradores ${ }^{59}$ engajados na funcionalização da superfície de nanopartículas de ouro via reação "click", estabeleceram que a melhor solubilidade dessas nanopartículas em solventes apolares era em uma mistura dioxano/hexano. Estes autores constataram que, tornando a mistura inicial mais apolar, ocorria um aumento concomitante da solubilidade e da conversão da azida em triazol. ${ }^{59,60}$ Já o uso de solventes polares como o metanol, DMF e terc-butanol é frequente em reações "click" envolvendo carboidratos, o que parece ser de grande importância para o alto rendimento das mesmas (Esquema 5). ${ }^{61}$

Outro exemplo interessante de como a mudança de solvente aumentou o rendimento e a velocidade de um tipo de reação "click" é representado pela síntese de derivados peptídicos macrocíclicos (Esquemas 6 e 7). As reações para obtenção do macrociclo 18 apresentaram-se muito lentas e com rendimentos muito baixos quando usados solventes como o tolueno, terc-butanol/água, MeCN/

\begin{tabular}{|c|c|c|c|c|c|c|c|}
\hline Fonte de metal & A. redutor & Solvente & Base & Temp. $\left({ }^{\circ} \mathrm{C}\right)$ & Tempo (h) & Rend. (\%) & Ref \\
\hline $\mathrm{CuCl} / \mathrm{Pd}_{2}(\mathrm{dba})_{3}$ & $\mathrm{Pd}(\mathrm{OEt})_{3}$ & dioxano & DIEA & 100 & $3-24$ & $20-63$ & 43 \\
\hline $\mathrm{CuBr}_{\mathrm{SMe}}$ & - & DMSO & DIEA & t.a. & 24 & 98 & 44 \\
\hline $\mathrm{CuI}$ & - & água & - & t.a. & $15-30$ & $52-92$ & 20 \\
\hline $\mathrm{CuI}$ & - & THF & DIEA & t.a. & 16 & $75-99$ & 5 \\
\hline $\mathrm{CuSO}_{4}$ & asc. sódio & água/terc-BuOH & - & t.a. & $12-24$ & $82-94$ & 6 \\
\hline $\mathrm{CuSO}_{4}$ & asc. sódio & água/ $\mathrm{CH}_{2} \mathrm{Cl}_{2}$ & - & t.a. & 24 & $80-98$ & 45 \\
\hline $\mathrm{Cu}(\mathrm{OAc})_{2}$ & - & terc-BuOH & - & t.a. & $<3$ & $76-99$ & 46 \\
\hline $\mathrm{CuBr}_{2}$ & asc. sódio & DMSO ou NMP & propilamina & t.a. & 12 & $63-78$ & 47 \\
\hline $\mathrm{Cu}(0) / \mathrm{Cu}_{2} \mathrm{O}$ & - & tolueno & - & t.a. & $2-4$ & $87-95$ & 48 \\
\hline CuOTf & $\left(\mathrm{N}_{2}\right)$ & tampão & bato* & t.a. & 16 & - & 49 \\
\hline $\mathrm{Cu} / \mathrm{C}$ & - & tolueno & TEA & 60 & $2-4$ & $92-99$ & 50 \\
\hline$\left[\mathrm{Cu}\left(\mathrm{CH}_{3} \mathrm{CN}\right)_{4}\right] \mathrm{PF}_{6}$ & - & $\mathrm{CH}_{2} \mathrm{Cl}_{2}$ ou água/ terc- $\mathrm{BuOH}$ & - & t.a. & 72 & 94 & 26 \\
\hline$\left[\mathrm{Cu}\left(\mathrm{CH}_{3} \mathrm{CN}\right)_{4}\right] \mathrm{OTf}$ & - & tampão & bato* & t.a. & 12 & $60-85$ & 27 \\
\hline
\end{tabular}
THF (Esquema 6). ${ }^{21}$

Tabela 1. Alguns exemplos de fontes de cobre para CuAAC 

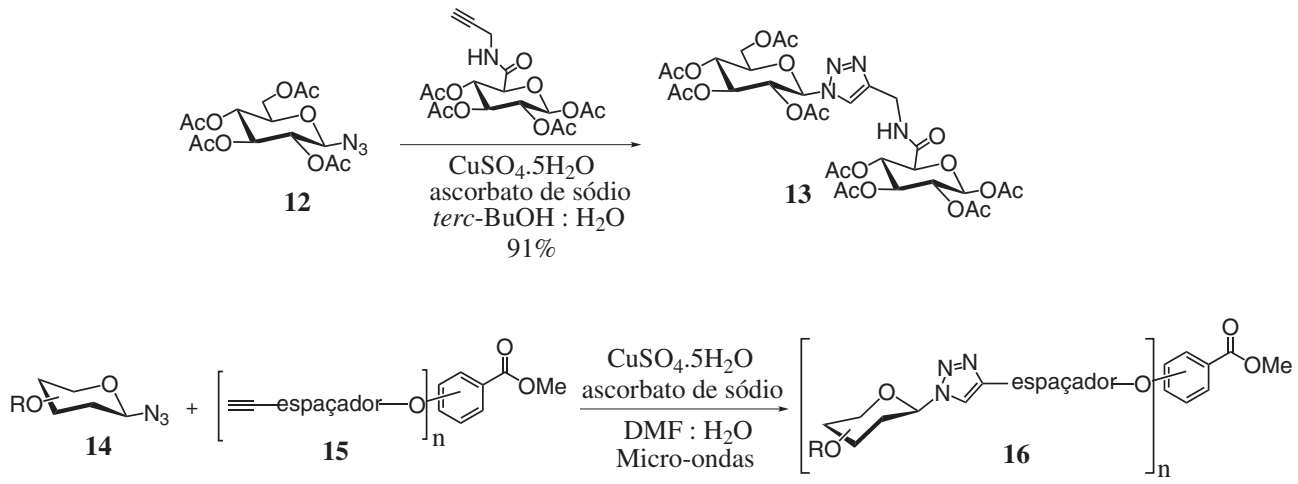

Esquema 5. Condições de reação comuns para a reação CuAAC de carboidratos

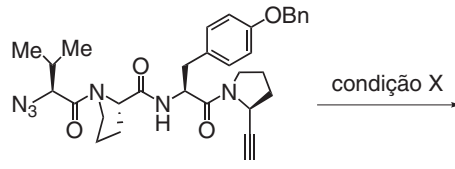

17

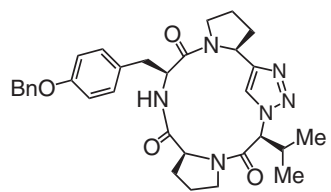

18

\begin{tabular}{ccc}
\hline condição X & tempo & rendimento \\
\hline $\mathrm{CuSO}_{4} /$ ascorbato/ & 8 dias & $0 \%$ \\
4:1 terc-BuOH: $\mathrm{H}_{2} \mathrm{O}$ & & \\
$\mathrm{Cul} / 2,6-$ Iutidina & 3 dias & $33 \%$ \\
5:1 MeCN:THF & dias & $43 \%$ \\
Cul/DBU/tolueno & 7 dias \\
\hline
\end{tabular}

Esquema 6. Reação CuAAC para obtenção de peptídeos macrocíclicos

Com o objetivo de otimizar este tipo de reação, Turner e colaboradores $^{62}$ utilizaram o $\mathrm{CuBr}$ como fonte de $\mathrm{Cu}(\mathrm{I})$, DIEA e 2,6-lutidina como bases/ligantes, e DMF como solvente para a ciclização do composto 19. Nestas condições, o macrociclo 20 (Esquema 7) foi obtido em menores tempos de reação e com altos rendimentos.
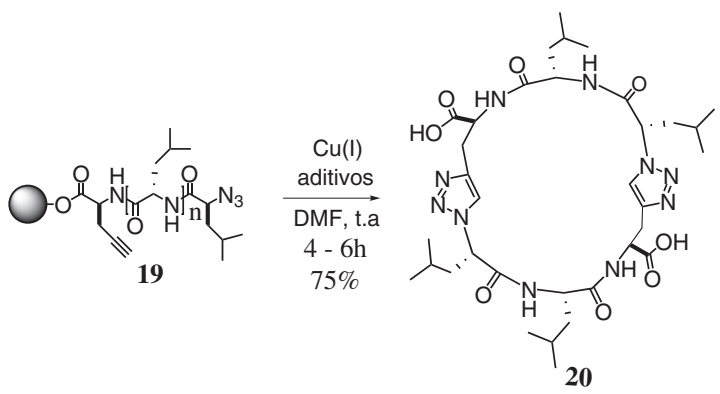

Esquema 7. Otimização de condições para obtenção de peptídeos macrocíclicos

\section{REATIVIDADE DOS SUBSTRATOS NA REAÇÃO “CLICK”}

O crescimento vertiginoso que a reação "click" vem experimentando em seu escopo e popularidade, desde 2002, gera uma necessidade de aprimorar o conhecimento sobre a mesma e um maior controle sobre o processo. ${ }^{63}$ A despeito dessa necessidade, investigações sistemáticas sobre os efeitos dos substituintes na reatividade dos substratos são raras. ${ }^{11,63}$ Os dados obtidos da literatura, até o presente, revelam que a reatividade das alquilazidas depende tanto das propriedades eletrônicas dos substituintes quanto do efeito estérico conferido por estes. De modo geral, as reações mostram-se mais rápidas quando as azidas envolvidas apresentam grupos retiradores de elétrons em sua estrutura e menor impedimento estérico. ${ }^{63,64}$
Azidas primárias e secundárias não apresentam grande diferença de reatividade para formação de triazóis, mas azidas terciárias não o fazem com facilidade, revelando a influência do fator estérico na coordenação terminal deste grupo com o cobre, para formação do estado de transição da reação.

Alcinos contendo grupos $\alpha$-carbonílicos são mais reativos que aqueles contendo apenas grupos alquila, ${ }^{65}$ os quais, por sua vez, têm reatividade similar ou pouco menor que a de alcinos aromáticos. $\mathrm{O}$ aumento do tamanho das cadeias tanto de alcinos quanto de azidas, como, por exemplo, nas reações de bioconjugação envolvendo proteínas e DNA, leva a um decréscimo da conversão dessas reações, revelando a importância de fatores estéricos, embora haja uma constante pesquisa e melhoria das condições para este tipo de reação. ${ }^{27}$

Outros grupos funcionais presentes nos solventes e substratos podem interferir na velocidade e no rendimento da reação. Como, por exemplo, substratos ou solventes contendo grupos nitrilas devem ser evitados uma vez que ocorre uma coordenação competitiva do grupo nitrila ao $\mathrm{Cu}(\mathrm{I})$ interferindo na formação do complexo alcino$\mathrm{Cu}(\mathrm{I})$. Atenção especial também deve ser dispensada às azidas alílicas substituídas do tipo 21 (Esquema 8), uma vez que estas são muito propensas ao rearranjo 1,3-sigmatrópico, podendo gerar misturas de produtos. ${ }^{66}$

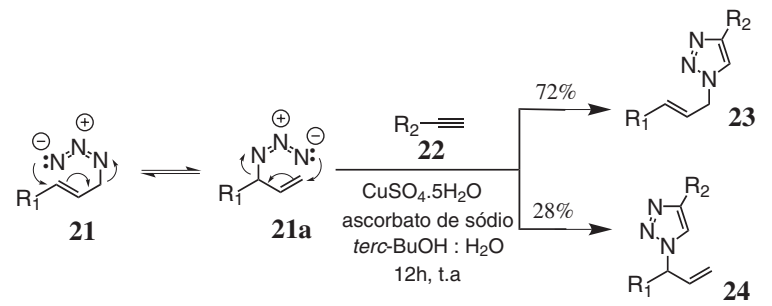

Esquema 8. Rearranjo sigmatrópico em azidas alílicas sob condições de reação "click"

\section{FORMAÇÃO DE SUBPRODUTOS NA REAÇÃO “CLICK”}

Apesar da reação "click" ter recebido considerável atenção por seus rendimentos virtualmente quantitativos e robustez, entre outros motivos, isto não descarta a existência de subprodutos em alguns experimentos. Dentre o espectro de subprodutos possíveis e já relatados na literatura, aqueles provenientes de acoplamentos oxidativos como, por exemplo, os triazolo-triazóis (composto 27, Esquema 9) são os mais comuns. ${ }^{6,11,67,68}$ A princípio, o ascorbato de sódio empregado na metodologia desenvolvida por Sharpless previne a formação dos triazolo-triazóis por fornecer de forma constante o $\mathrm{Cu}(\mathrm{I})$ e evitar a formação de $\mathrm{Cu}$ (II). Entretanto, a ausência de um redutor no meio pode fazer com que o oxigênio favoreça a formação dos subprodutos. Os triazolo-triazóis podem ser favorecidos também pela presença 


$$
25+{ }_{26}^{+}{ }_{N^{N}}^{\oplus} N^{N-R_{2}} \frac{\text { catálise por cobre }}{\text { base }}
$$

Esquema 9. Condições de favorecimento dos subprodutos triazolo-triazóis

de bases como $\mathrm{K}_{2} \mathrm{CO}_{3}$, di-isopropiletilamina (DIPEA), 2,6-lutidina, trietilamina (TEA) ou piridina. ${ }^{6,67,68}$

A utilização de base na reação "click", além de propiciar a formação dos triazolo-triazóis, também pode favorecer o aparecimento de triazóis trissubstituídos com resíduo de alcinos em C-5 (composto 31, Esquema 10). Esses produtos secundários são importantes quando as bases utilizadas são $\mathrm{LiOH}, \mathrm{K}_{2} \mathrm{CO}_{3}$ e, especialmente, $\mathrm{KOH}$. Além da forte influência da base, outros fatores propiciam a formação deste tipo de subproduto como, por exemplo, o uso do $\mathrm{Cu}\left(\mathrm{CH}_{3} \mathrm{CN}\right)_{4} \mathrm{PF}_{6}$ como fonte de $\mathrm{Cu}(\mathrm{I})$, o oxigênio e o uso de ligante diamina. ${ }^{68,69}$

Como citado anteriormente, dentre as várias etapas que compõem o mecanismo proposto e aceito para a reação "click", a última etapa corresponde a uma protonação do derivado triazólico que está ligado diretamente ao átomo de cobre. ${ }^{16,21}$ Outros eletrófilos que porventura estejam no meio reagente podem exercer o papel do próton, gerando subprodutos 1,2,3-triazóis-1,4,5-trissubstituídos como, por exemplo os triazóis contendo átomo de iodo na posição 5. ${ }^{28,70-72} \mathrm{~A}$ adição de iodo na posição 5 tem sido obtida com sucesso com o uso de $\mathrm{ICl}$ ou NBS conjuntamente com o catalisador $\mathrm{CuI}$ (Esquema 11)..$^{71,73}$

$\mathrm{O}$ ácido alquinoico e seus derivados, sob condição de reação CuAAC, também levou ao aparecimento de subprodutos: as enóis lactonas (composto 38, Esquema 12) e seus correspondentes produtos de hidrólise (composto 39, Esquema 12). Para a formação destes produtos, o grupo azida não participa da reação, mas ocorre apenas a simples atuação do $\mathrm{Cu}(\mathrm{I})$ sobre o substrato derivado do ácido alquinoico. Os dados experimentais confirmaram a reprodutibilidade na obtenção dos subprodutos enóis lactonas numa infinidade de substratos derivados do ácido alquinoico. ${ }^{74}$

Além destes subprodutos mostrados anteriormente, já foram relatadas, nas condições da reação CuAAC, a formação de amidinas, imidatos e amidas quando se utilizou como material de partida sulfonilazidas ${ }^{75}$ de alcoóis e amidas utilizando-se alcinos $\alpha$ - $\operatorname{carbamatos}^{76} \mathrm{e}$ de dímeros quando se utilizaram inamidas como alcinos de partida. ${ }^{77-79}$ Adicionalmente, em algumas reações $\mathrm{CuAAC}$ ainda existe a possibilidade de formação de compostos como diacetiletos e 5-hidroxitriazóis, que pode ser minimizada com o uso de 2,6-lutidina e a exclusão do oxigênio do meio reagente. ${ }^{6}$

\section{APLICAÇÕES DA REAÇÃO “CLICK”}

Uma das primeiras aplicações da reação CuAAC foi publicada há cerca de 9 anos por Meldal e colaboradores, ${ }^{5}$ que usaram a reação para sintetizar peptidotriazóis em suporte sólido. Inicialmente, peptídeos ligados a uma resina de PEGA $_{800}$ foram funcionalizados com um alcino, na forma de grupos $N$-propinoíla terminal ou propargilglicina. Em seguida, uma grande variedade de azidas derivadas de açúcares, aminoácidos e aminoalcoóis protegidos foi acoplada aos peptídeos. Essas reações foram realizadas à temperatura ambiente, em THF, e como fonte de cobre foi usado CuI na presença de DIPEA (N,N-di-isopropiletilamina), levando à formação de 1,2,3-triazóis em bons rendimentos como, por exemplo, os compostos 40 e 41 (Figura 2).

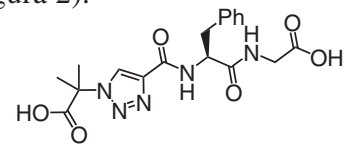

40

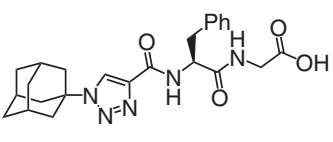

41
Figura 2. Peptidotriazóis obtidos via CuAAC em fase sólida

Após o sucesso obtido por este grupo de pesquisa na síntese de 1,2,3-triazóis 1,4-dissubstituídos, as cicloadições entre alcinos e azidas catalisadas por $\mathrm{Cu}(\mathrm{I})$ passaram a ser amplamente utilizadas nas mais diversas áreas de pesquisa, incluindo química medicinal, ${ }^{25,80-96}$ bioconjugação, ${ }^{31,42,97-103}$ química de materiais, ${ }^{38,104-129}$ química supramolecular, ${ }^{130-143}$ síntese de catalisadores, ${ }^{144-148}$ dentre outras.

\section{Química medicinal}

A reação "click" é uma importante ferramenta para a descoberta e desenvolvimento de novos fármacos, além de possibilitar modificações daqueles já existentes, visando à melhoria das suas propriedades farmacológicas e farmacocinéticas.

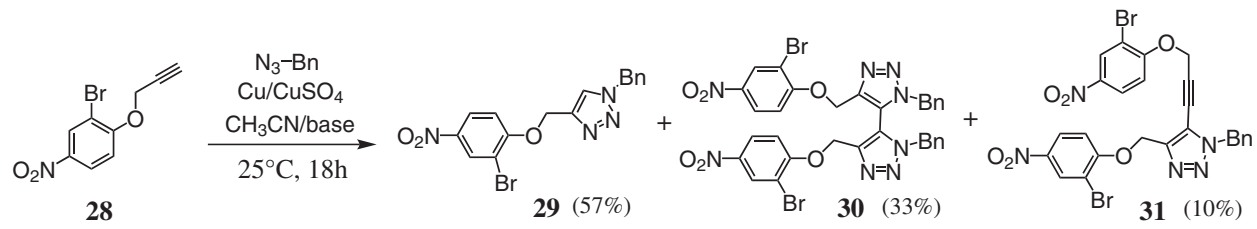

Esquema 10. Formação de subproduto do tipo alcino substituído
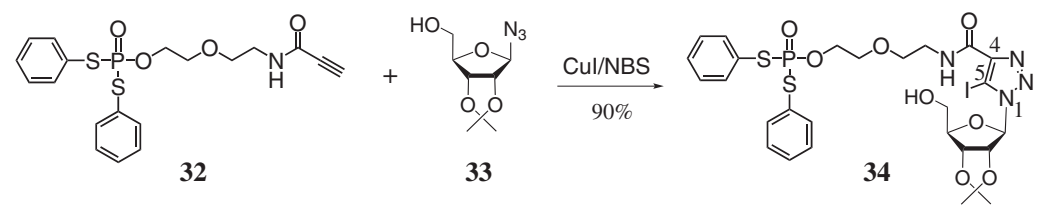

Esquema 11. Formação de 5-iodotriazóis

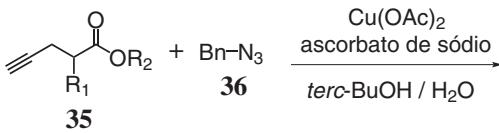

35<smiles>[R7]C([18F])C(=O)O[18F]</smiles>

37

$32-96 \%$

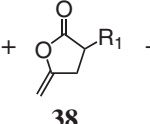

$0-48 \%$

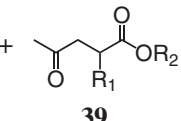

39

$0-35 \%$

Esquema 12. Enóis-lactonas como subprodutos obtidos em condição de reação "click" com ácidos alquinoicos e derivados 
O 1,2,3-triazol é relevante para a química medicinal, pois pode não só atuar como um grupo farmacofórico,$^{80,149,150}$ mas também como uma conexão entre duas ou mais substâncias de interesse, em uma estratégia de hibridação molecular, por exemplo. Além disso, o anel triazólico funciona como um bioisóstero do grupo amida por apresentar propriedades físico-químicas semelhantes. A unidade triazólica atua como uma conexão rígida, que acomoda os grupos $\mathrm{R}_{1}$ e $\mathrm{R}_{2}$ (Figura 3), a uma distancia de 5,0 A (por comparação, a distância dos mesmos grupos na amida é de $3,8 \AA$ ). Em contraste com as amidas, o anel triazólico é mais estável, já que não sofre hidrólise, oxidação ou redução. 1,2,3-triazóis possuem um grande momento de dipolo ( 5 D) e os nitrogênios das posições 2 e 3 atuam como fracos aceptores de ligação de hidrogênio. . $^{14,151}$

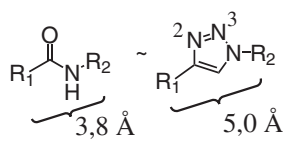

Figura 3. 1,2,3-triazol: bioisóstero de amidas

Assim, alguns grupos de pesquisa focam seus estudos na substituição de amidas por anéis triazólicos em moléculas onde as ligações amídicas são cruciais para a atividade biológica. Marzo e colaboradores, ${ }^{81}$ por exemplo, sintetizaram análogos de capsaicinoides (Figura 4), trocando ligações amídicas por anéis triazólicos, para verificar se haveria alguma alteração na atividade biológica. Os capsaicinoides triazólicos apresentaram atividade comparável à daqueles naturais, o que comprova o bioisosterismo destes grupos.

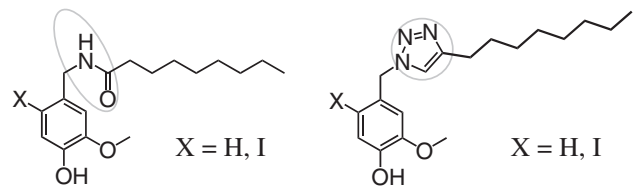

Figura 4. Capsaicinoides e seus análogos triazólicos

Guarna e colaboradores ${ }^{25}$ sintetizaram uma sequência tripeptido mimética da arginina-glicina-ácido aspártico com potencial aplicação como agente antiangiogênico em tumores com superexpressão de integrinas (Esquema 13). Neste exemplo, o anel triazólico foi usado para mimetizar a glicina, atuando como um espaçador. Dentre os compostos sintetizados, o derivado $\mathbf{4 2}$ apresentou atividade biológica relevante sobre receptores integrinas associados com a membrana plasmática de células tumorais, o que o torna um promissor agente antiangiogênico.

A reação "click" tem sido também empregada na modificação de produtos naturais bioativos visando à melhora da atividade biológica destes. ${ }^{152,153}$ Como exemplo, há o trabalho de Genazzani e colabora- dores ${ }^{82}$ que sintetizaram análogos do resveratrol, um produto natural que possui diversas atividades biológicas (Figura 5). Alguns destes análogos, contendo um anel triazólico, apresentaram atividade citotóxica e antiproliferativa maior do que o protótipo natural. Este anel funcionou como um bioisóstero da ligação dupla.
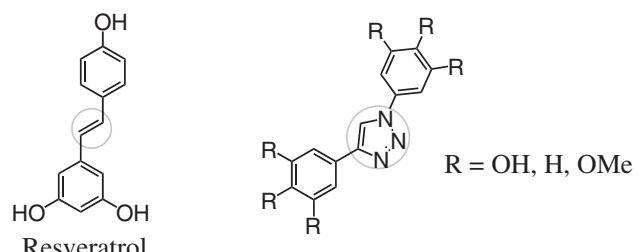

Figura 5. Resveratrol e seus análogos modificados com anel triazólico

Silva Jr. e colaboradores ${ }^{83}$ também usaram a CuAAC para modificar produtos naturais, sintetizando derivados da nor- $\beta$-lapachona (Esquema 14). Este produto natural apresenta algumas atividades biológicas, entre as quais, citotóxica e tripanossomicida. A partir da nor- $\beta$-lapachona, em três etapas, foram obtidos triazóis naftoquinoidais como, por exemplo, o derivado 43 (Esquema 14). Todos os derivados sintetizados apresentaram atividade tripanossomicida maior do que a do precursor, sendo o derivado 43 o mais ativo.

Vários outros exemplos de síntese de substâncias com atividade biológica construídas utilizando a CuAAC podem ser encontrados na literatura como, por exemplo, compostos com atividades anticâncer, ${ }^{84,85}$ antiprotozoária, ${ }^{86,87}$ citotóxica, ${ }^{88}$ antimicobacteriana, ${ }^{89}$ antifúngica, ${ }^{90}$ antimalárica, ${ }^{91}$ além de diversos inibidores enzimáticos, como os da enzima proteína tirosina fosfatase $1 \mathrm{~B},{ }^{92}$ da enzima glucocerebrosidase, relacionados com a doença de Gaucher, ${ }^{93}$ da protease do HIV ${ }^{80}$ de neuraminidases $;{ }^{94}$ da enzima nicotinamida fosforribosiltransferase, associados à atividade antitumoral, ${ }^{95} \mathrm{da}$ enzima $\alpha$-glucosidase, associada à diabetes mellitus tipo II, ${ }^{154}$ dentre outros.

A reação "click" apresenta também grande contribuição para a química combinatória, que é uma promissora ferramenta para a descoberta e o desenvolvimento de novos fármacos. Como se sabe, nesta metodologia de síntese os produtos são formados simultaneamente e podem ser testados biologicamente de uma só vez (in situ). O principal objetivo desta estratégia é reduzir o tempo necessário para a obtenção de um novo fármaco. A síntese de quimiotecas pode ser realizada através da utilização de polímeros insolúveis, solúveis ou pela tradicional síntese em solução. Um exemplo da aplicação da CuAAC em química combinatória pode ser visto na triagem in situ de inibidores de protease do vírus HIV, realizada por Sharpless e colaboradores. ${ }^{155}$ Neste estudo, duas azidas ( 44 e 46, Esquema 15) derivadas do amprenavir, um fármaco antirretroviral, foram colocadas, separadamente, em placas contendo 96 cavidades. Em seguida, uma

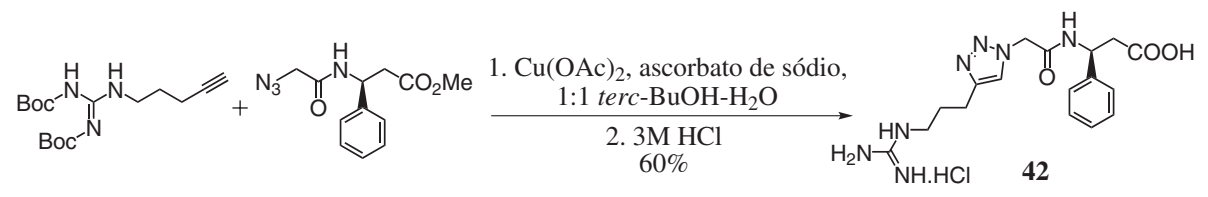

Esquema 13. Síntese do agente antiangiogênico 42

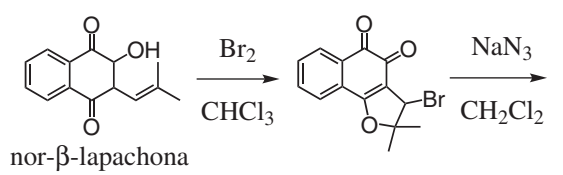

nor- $\beta$-lapachona

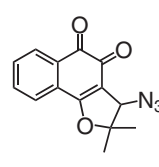

$\mathrm{N}_{3}$
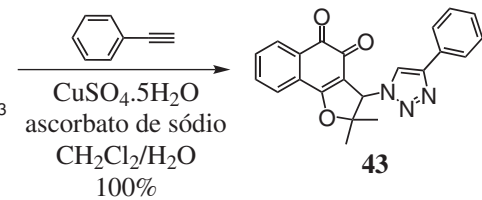

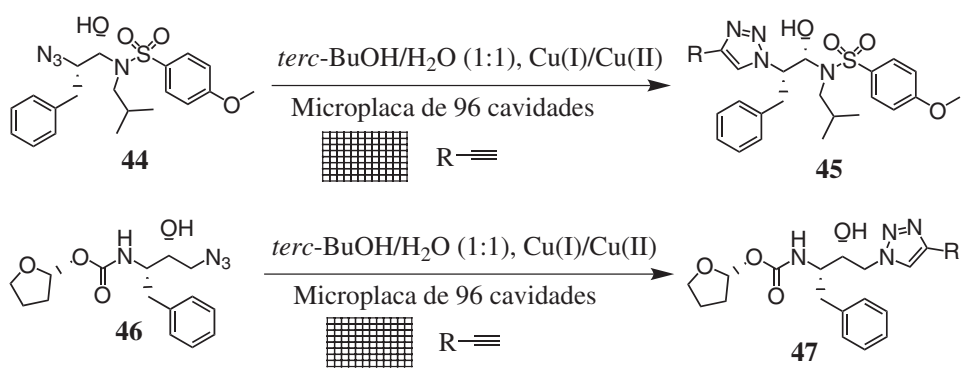

Esquema 15. Uso da química combinatória na triagem de inibidores de protease do HIV

série de alcinos foi colocada para reagir com as azidas, originando derivados triazólicos, que foram testados in situ contra proteases do vírus HIV. Apenas os derivados triazólicos oriundos da azida $\mathbf{4 4}$ apresentaram forte inibição da enzima. Em um estudo posterior de Wong e colaboradores, ${ }^{156}$ concluiu-se que o anel triazólico atua como um substituto efetivo da ligação amídica.

Outros exemplos de aplicação da reação "click" em química combinatória podem ser encontrados na literatura. ${ }^{157-159}$

\section{Bioconjugação}

Como já mencionado, a reação CuAAC tornou-se muito popular em áreas de interface com a química orgânica. A bioconjugação representa a interface entre a biologia molecular e a química orgânica e se refere à derivatização de biomoléculas, originando um novo composto que une as propriedades dos componentes iniciais. ${ }^{160,161}$ Biomoléculas tais como peptídeos, proteínas, DNA, ácidos nucleicos, oligossacarídeos ou glicoconjugados são extremamente úteis no estudo dos sistemas biológicos. No entanto, a presença de diversos grupos funcionais nestas estruturas restringe o número de reações que podem ser utilizadas para quaisquer modificações das mesmas. Assim, a estratégia ideal é empregar reações que sejam altamente seletivas, que possam ser realizadas em solvente aquoso ou orgânico, que conduzam a produtos com altos rendimentos e que sejam ortogonais aos demais grupos funcionais. Neste contexto, a reação "click" torna-se ideal para aplicação em bioconjugação.

Finn e colaboradores ${ }^{97}$ foram os primeiros a utilizar a $\mathrm{CuAAC}$ para a bioconjugação. Neste trabalho, o capsídeo do vírus mosaico do caupi foi ligado a moléculas de fluoresceína via reação "click", em meio aquoso. O capsídeo do vírus se assemelha a uma gaiola, formada por 60 cópias idênticas de duas unidades de proteínas assimétricas. Após a funcionalização dos resíduos terminais dessas proteínas com alcino ou azida, realizou-se a cicloadição catalisada por $\mathrm{Cu}(\mathrm{I}) \mathrm{com}$ o derivado da fluoresceína devidamente funcionalizado (alcino ou azida) (Figura 6).
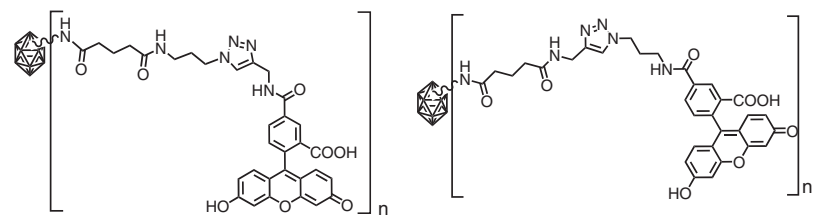

Figura 6. Bioconjugados do capsídeo do vírus mosaico do caupi com fluoresceína

Uma preocupação por parte de alguns autores quanto ao uso de cobre na catálise de reações $\mathrm{CuAAC}$ envolvidas em meio biológico advém da possível toxicidade deste metal. ${ }^{162}$ Assim, na busca por um método alternativo e não invasivo de imagem de biomoléculas em organismos vivos, Wu e colaboradores ${ }^{42}$ relataram o uso do ligante BTTES (tris(triazoilmetil)amina), o qual complexa com o $\mathrm{Cu}(\mathrm{I})$ e o estabiliza, evitando que o $\mathrm{Cu}(\mathrm{I})$ livre no organismo gere espécies reativas de oxigênio e nitrogênio. Com o uso do ligante BTTES foram obtidos bioconjugados de linfócito $\mathrm{T}$ e glicanos, que foram utilizados para fazer imagens in vivo de glicanos. Estes bioconjugados foram obtidos a partir da reação "click" entre resíduos de alcino ou azida do ácido siálico presente na superfície celular e azida ou alcino-biotina, respectivamente.

Além destes exemplos, várias outras modificações de biomoléculas foram realizadas, tais como a incorporação de fluoróforos em proteínas ou nucleosídeos; ${ }^{98-100}$ de radioisótopos em peptídeos ou nucleosídeos na síntese de radiotraçadores; ${ }^{101,102}$ de ferrocenos em aminoácidos ou peptídeos ${ }^{103}$ e, de oligossacarídeos em peptídeos na síntese de vacinas. ${ }^{31}$

\section{Química de materiais}

Na química de materiais, a reação "click" vem sendo extensivamente utilizada na obtenção de diversos materiais, tais como dendrímeros, ${ }^{37,38,104-107}$ polímeros, ${ }^{108-111}$ fases estacionárias modificadas, ${ }^{119-122}$ organogéis, ${ }^{123-125}$ macrociclos, ${ }^{134-136}$ dentre outros.

Dendrímeros são moléculas formadas a partir de um núcleo polifuncionalizado através do crescimento radial de camadas, de forma repetitiva. A molécula gerada após a incorporação da primeira camada de unidades monoméricas é designada dendrímero de primeira geração. A incorporação de sucessivas camadas leva à formação de polímeros de segunda geração, terceira e assim por diante. A natureza e o número de camadas podem ser controlados por um planejamento da síntese. Os maiores problemas encontrados nestas sínteses estão relacionados às dificuldades de purificação, necessidade do uso de excesso dos reagentes e lentidão das separações cromatográficas. Desta forma, a reação "click" torna-se muito útil na síntese de dendrímeros, visto que ela é capaz de contornar estes problemas, por ser muito eficiente, praticamente não gerar subprodutos, necessitar somente de quantidades estequiométricas dos reagentes, além de apresentar excelentes rendimentos. ${ }^{163,164}$

Os dendrímeros recebem muita atenção devido às suas propriedades únicas e aplicações em química medicinal e de materiais. Fokin e colaboradores ${ }^{37}$ foram os pioneiros no uso da reação "click" na síntese de dendrímeros utilizando uma síntese convergente. Obtiveram o dendrímero 49 a partir do núcleo 48 (Esquema 16), envolvendo CuAAC. Cabe ressaltar que os dendrímeros obtidos por este método foram mais facilmente purificados do que aqueles obtidos pelos métodos clássicos, e com melhores rendimentos.

Uma aplicação mais recente na síntese de dendrímeros foi descrita por Zhang e colaboradores, ${ }^{104}$ na qual o dendrímero 50 (Figura 7), contendo grupos carbazólicos, foi sintetizado utilizando a reação "click". Este dendrímero apresentou uso promissor em dispositivos eletrofosforecentes, sendo sua atividade testada em um dispositivo de diodo orgânico emissor de luz (OLED).

Além destes exemplos, vários outros dendrímeros com diversas aplicações foram sintetizados..$^{38,105-107}$ 


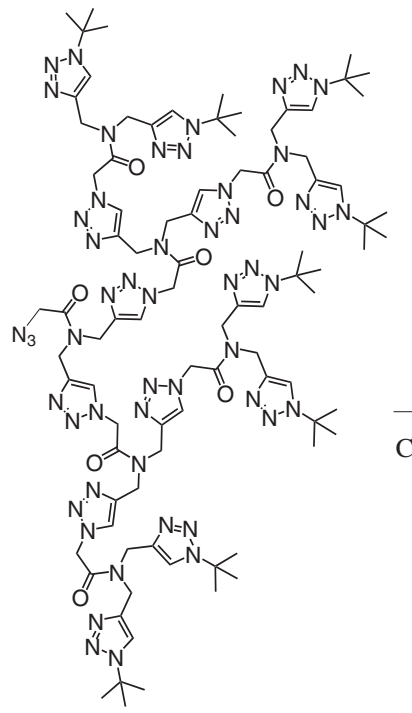

Esquema 16. Dendrímero 47 obtido via CuAAC

A reação “click” também é largamente empregada na obtenção de outros materiais como, por exemplo, polímeros, ${ }^{108-111}$ organogéis, ${ }^{123-125}$ colas/adesivos, ${ }^{112,113}$ fios moleculares, ${ }^{114}$ biossensores, ${ }^{115}$ quimiossensores, ${ }^{116}$ radiotraçadores, ${ }^{117}$ dentre outros.

Polímeros conjugados têm sido largamente explorados pela potencial aplicação em dispositivos optoeletrônicos, ${ }^{165}$ diodos, ${ }^{166}$ lasers orgânicos, ${ }^{167}$ transistores de película fina ${ }^{168}$ e dispositivos ópticos não lineares. ${ }^{169}$ Reek e colaboradores ${ }^{118}$ sintetizaram alguns polímeros conjugados por meio da reação "click", por exemplo 51 e 52 (Figura 8). Devido às propriedades luminescentes apre-

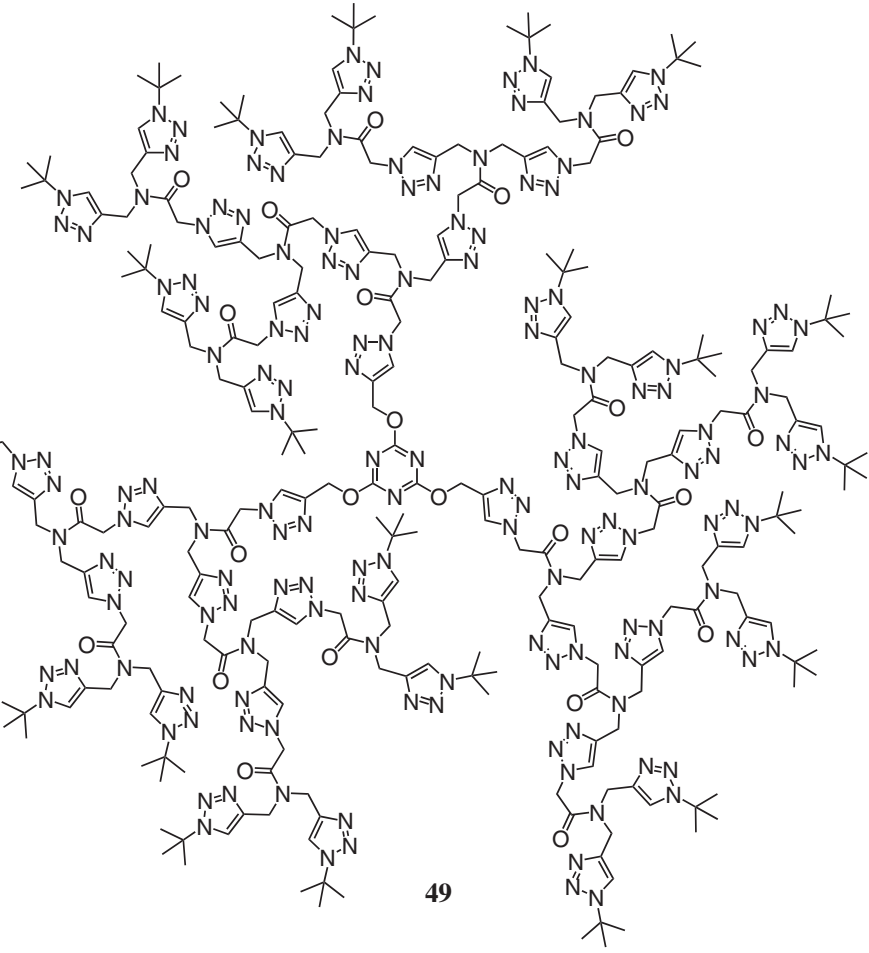

sentadas por estes polímeros, são candidatos a uso em materiais fosforescentes.

Outra aplicação da reação "click" é a modificação de fases estacionárias de colunas cromatográficas. Um exemplo interessante é a pesquisa de Liang e colaboradores, ${ }^{119}$ que modificaram fases estacionárias para cromatografia líquida de alta eficiência (CLAE) e cromatografia líquida de interação hidrofílica (HILIC). Este trabalho envolveu a reação "click" entre 3-azidopropil trietoxissilano e alcinos terminais ligados a grupos apolares (originando colunas de fase reversa - 53) ou polares (originando colunas para HILIC - 54)

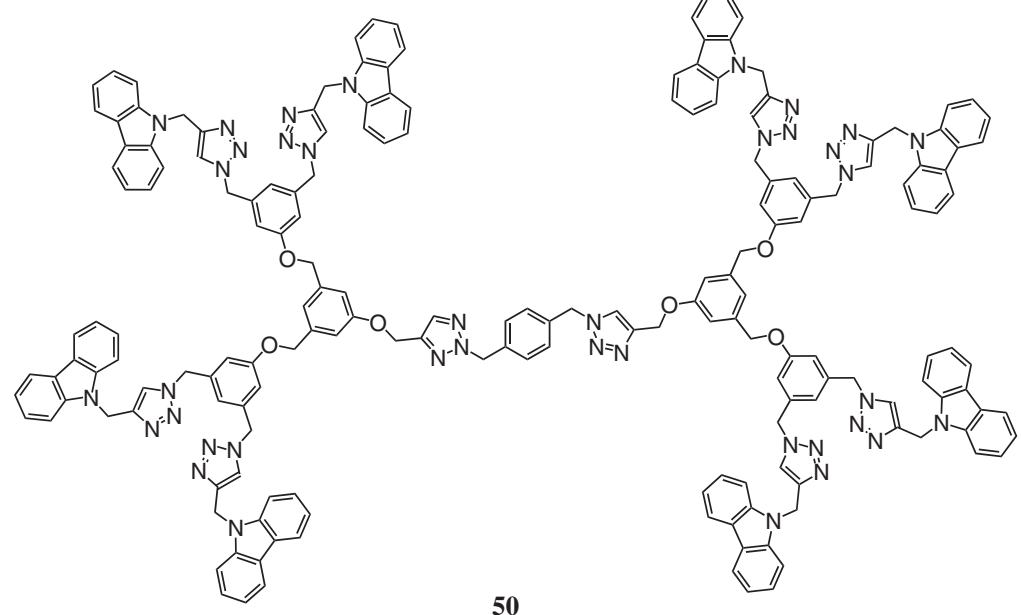

Figura 7. Dendrímero 48 obtido via CuAAC
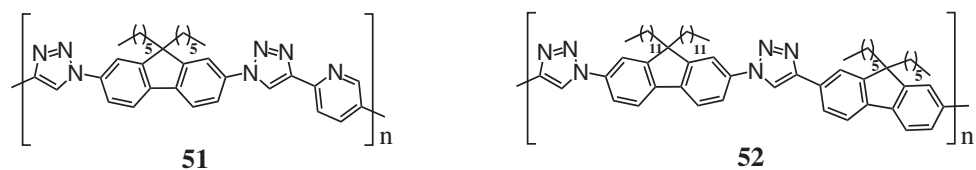

52

Figura 8. Polímeros conjugados com propriedades luminescentes 
(Figura 9). Outros exemplos desta aplicação podem ser encontrados na literatura. ${ }^{119-121}$
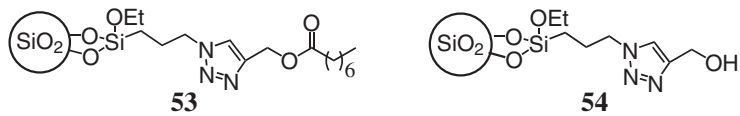

Figura 9. Fases estacionárias para cromatografia preparadas via CuAAC

Organogéis são estruturas altamente definidas, organizadas por interações supramoleculares entre moléculas orgânicas pequenas e médias ou micelas poliméricas. Eles apresentam várias aplicações, desde o transporte de fármacos até o uso como suporte de engenharia de tecidos. ${ }^{170}$ A reação "click" já foi usada na formação de ligações cruzadas de hidrogéis por alguns grupos de pesquisa. ${ }^{123,124}$ Fin e colaboradores, ${ }^{125}$ por exemplo, utilizaram a reação "click" para gerar ligações cruzadas entre organogéis, que são interações bem mais significativas do que apenas as interações supramoleculares presentes no material inicial (Esquema 17).

A criação de sistemas fotossintéticos artificiais é de grande importância para o entendimento dos processos bioenergéticos e para o desenvolvimento de dispositivos fotoquímicos e aparelhos optoeletrônicos. Baseado nisso, um mimetizador eficiente e funcional de complexos fotossintéticos do tipo antena foi desenvolvido e sintetizado por $\mathrm{Ng}$ e colaboradores. ${ }^{126} \mathrm{O}$ complexo 55 é constituído de um núcleo de zinco porfirina (II) conectado a uma molécula de $\mathrm{C}_{60}$ e três unidades de dipirrometeno, sendo que estas últimas foram acopladas via reação "click" (Esquema 18).

Mais aplicações da $\mathrm{CuAAC}$ na química de nanomateriais podem ser encontradas na literatura. ${ }^{127-129}$

\section{Química supramolecular}

Outra área em que a reação "click" vem sendo muito aplicada é na química supramolecular, a qual se baseia nas interações não covalentes entre moléculas. Os calixarenos se incluem neste contexto e têm sido modificados via reação "click" com o objetivo principal de aumentar a solubilidade dos mesmos, como pode ser verificado no trabalho de Ryu e Zhao (Esquema 19). ${ }^{130}$ A CuAAC também está envolvida em outras modificações de calixarenos. ${ }^{131-133}$

Os macrociclos também são importantes blocos construtores em química supramolecular, devido às suas diversas aplicações como cavidades moleculares e receptores artificiais. As reações de macrociclização de estruturas lineares contendo uma função azida e outra alcino são altamente dependentes da probabilidade de encontro dos dois grupos reativos envolvidos na ciclização. ${ }^{11} \mathrm{O}$ primeiro peptídeo mimético cíclico obtido via reação CuAAC foi sintetizado por Meldal e colaboradores (Figura 10). ${ }^{134} \mathrm{~A}$ obtenção deste macrociclo foi feita em suporte sólido, em alto rendimento e pureza. O anel triazólico, neste caso, foi usado com a estratégia de mimetizar uma ponte de dissulfeto.

Ciclodextrinas são macrociclos formados por seis, sete ou oito subunidades de glicopiranose, designados como $\alpha, \beta$ ou $\gamma$ ciclodextrinas, respectivamente. Possuem uso difundido na química supramolecular, pois são capazes de formar compostos de inclusão com moléculas hidrofóbicas, o que permite que eles sejam carreados em solventes polares. Gin e colaboradores ${ }^{135}$ sintetizaram análogos de ciclodextrina via ciclodimerização de um trissacarídeo com a função alcino em uma extremidade e azido na outra, por meio da reação "click". O ciclodímero obtido (composto 56, Esquema 20) exibiu um perfil de associação ao 8-anilino-1-naftalenossulfonato<smiles>C#CCCC(=O)NC1(NC(=O)CNC=C)CCCC2CCCC2NC1=O</smiles>

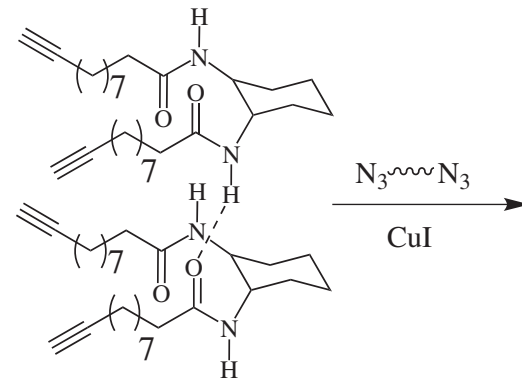

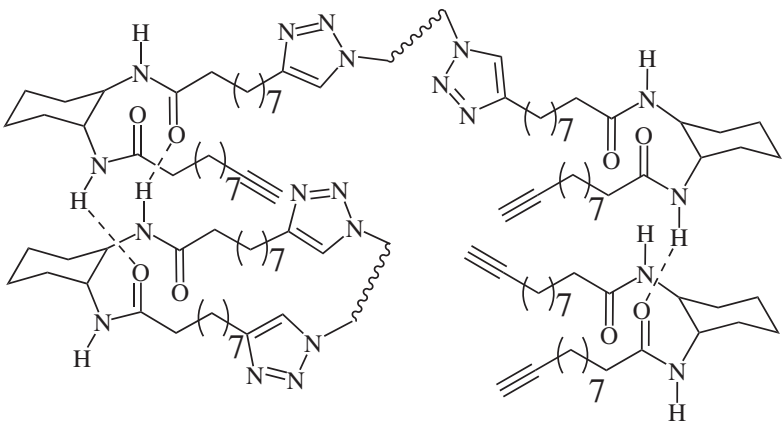

Esquema 17. Formação de ligações cruzadas de organogéis utilizando CuAAC
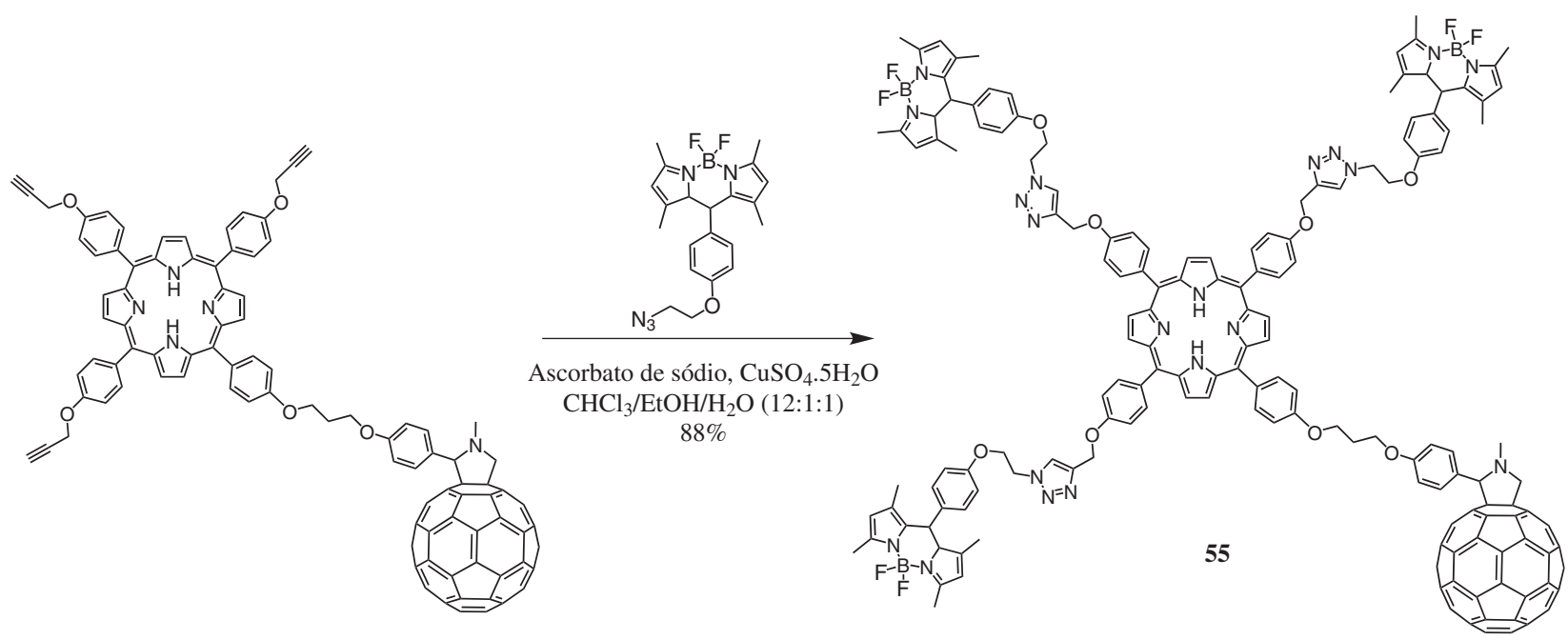

Esquema 18. Obtenção do complexo 53 utilizando CuAAC 


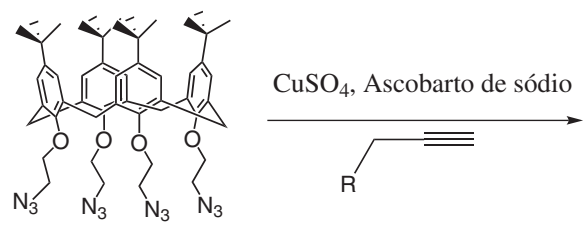

Esquema 19. Preparação de calixarenos hidrossolúveis

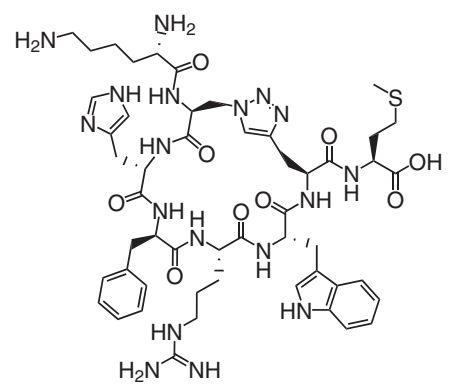

Figura 10. Peptídeo mimético cíclico obtido via CuAAC

similar àquele apresentado pela $\beta$-ciclodextrina. Em 2005, este mesmo grupo de pesquisadores sintetizou um ciclotrímero e um ciclodímero utilizando a mesma metodologia, a partir de um monossacarídeo e de um dissacarídeo, respectivamente. ${ }^{136}$
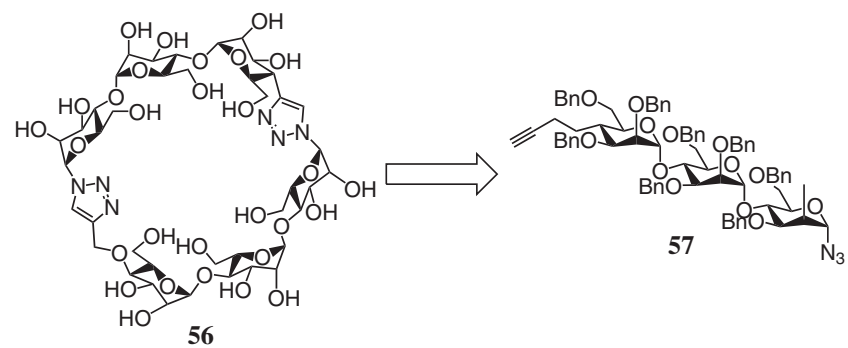

Esquema 20. Macrociclo análogo à $\beta$-ciclodextrina

Ainda na química supramolecular, a reação "click" pode ser aplicada na síntese de rotaxanos, ${ }^{137-139}$ catenanos, ${ }^{140-142}$ e nanocavidades. ${ }^{143}$

\section{Catalisadores}

A reação "click" tem sido aplicada também na obtenção de catalisadores. Liang e colaboradores ${ }^{144}$ sintetizaram uma série de dendrímeros quirais contendo triazóis obtidos via $\mathrm{CuAAC}$, que atuaram como catalisadores na redução assimétrica de cetonas pró-quirais usando boranos (Esquema 21).

Outros exemplos de catálise utilizando ligantes sintetizados a partir da reação "click" são relatados como, por exemplo, catalisadores em reações de acoplamento do tipo Suzuki-Miyaura; ${ }^{145}$ catalisadores que promovem reação de alquilação alílica regiosseletiva; ${ }^{146}$ catalisadores que promovem benzoilação enantiosseletiva de dióis ${ }^{147} \mathrm{e}$ catalisadores que promovem hidrogenação assimétrica. ${ }^{148}$

\section{CONSIDERAÇÕES FINAIS}

A velocidade com que pesquisadores de várias áreas adotam uma nova metodologia que surge na literatura é um claro indicador da utilidade e versatilidade da mesma. Neste contexto, a reação de formação de 1,2,3-triazóis a partir de alcinos e azidas, usando cobre como catalisador, tem se mostrado uma das mais eficazes formas para se conectar covalentemente duas moléculas contendo vários grupos
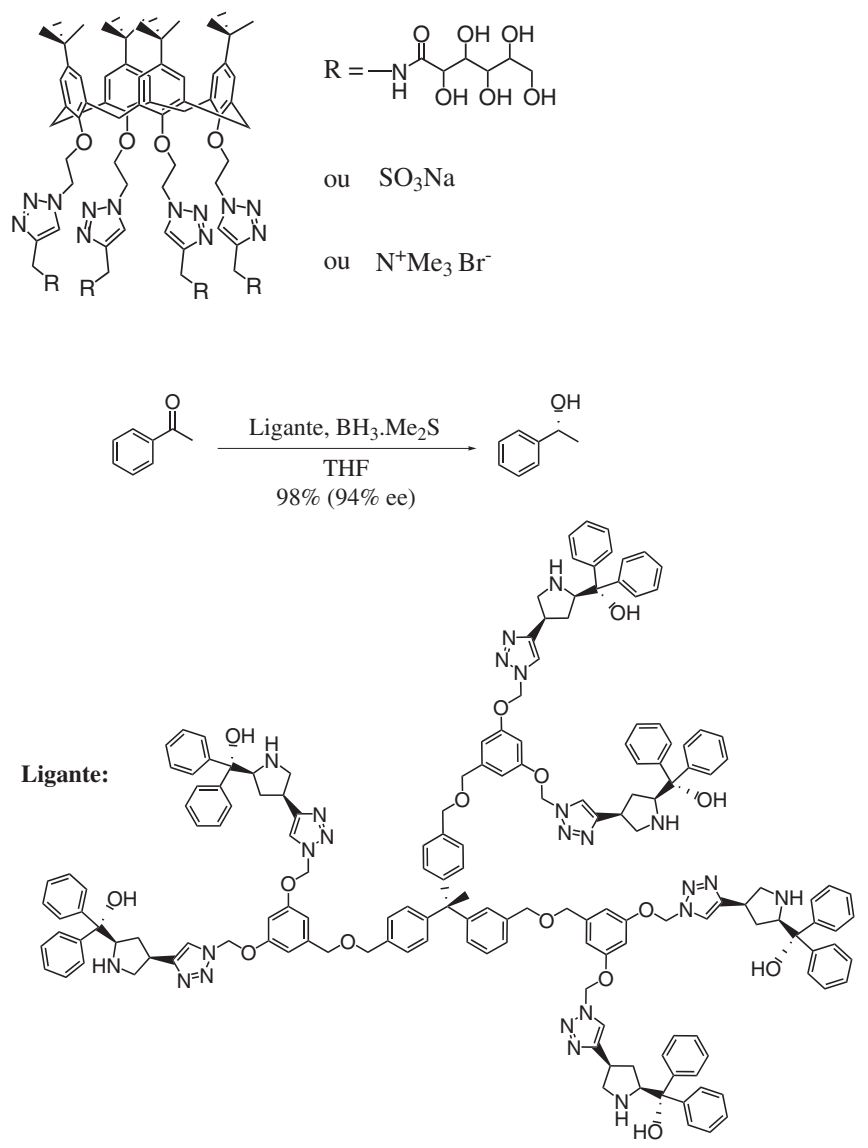

Esquema 21. Redução enantiosseletiva de cetona pró-quiral

funcionais. Como o desenvolvimento da ciência contemporânea está diretamente relacionado à praticidade de se obter novas e variadas moléculas, a reação $\mathrm{CuAAC}$ deverá, provavelmente, continuar a ser cada vez mais utilizada e estudada por grupos de pesquisa em todo mundo. No Brasil, ao nosso conhecimento, vários grupos já publicaram trabalhos nesta área, ${ }^{14,45,83,87-89,96,129,154}$ a maioria visando a síntese de moléculas bioativas. Apesar disso, acreditamos que ainda haja muito a ser explorado no país, tanto na área de química medicinal quanto na área de materiais e de produtos naturais (semissíntese).

\section{REFERÊNCIAS}

1. Kolb, H. C.; Finn, M. G.; Sharpless, K. B.; Angew. Chem., Int. Ed. 2001, 40, 2004.

2. Melo, J. O. F.; Donnici, C. L.; Augusti, R.; Ferreira, V. F.; Souza, M. C. B. V.; Ferreira, M. L. G.; Cunha, A. C.; Quim. Nova 2006, 29, 569.

3. Michael, A.; J. Prakt. Chem. 1893, 46, 94.

4. Huisgen, R.; Szeimies, G.; Moebius, L.; Chem. Ber. 1967, 100, 2494.

5. Tornфe, C. W.; Christensen, C.; Meldal, M.; J. Org. Chem. 2002, 67, 3057.

6. Rostovtsev, V. V.; Green, L. G.; Fokin, V. V.; Sharpless, K. B.; Angew. Chem., Int. Ed. 2002, 41, 2596.

7. Lundberg, P.; Hawker, C. J.; Hult, A.; Malkoch, M.; Macromol. Rapid Commun. 2008, 29, 998.

8. Buckley, B. R.; Dann, S. E.; Harris, D. P.; Heaney, H.; Stubbs, E. C.; Chem. Commun. 2010, 46, 2274.

9. Punna, S.; Kuzelka, J.; Wang, Q.; Finn, M. G.; Angew. Chem., Int. Ed. 2005, 44, 2215

10. Ahlquist, M.; Fokin, V. V.; Organometallics 2007, 26, 4389.

11. Meldal, M.; Tornфe, C. W.; Chem. Rev. 2008, 108, 2952.

12. Hein, J. E.; Fokin, V. V.; Chem. Soc. Rev. 2010, 39, 1302. 
13. Himo, F.; Lovell, T.; Hilgraf, R.; Rostovtsev, V. V.; Noodleman, L.; Sharpless, K. B.; Fokin, V. V.; J. Am. Chem. Soc. 2005, 127, 210.

14. Aragão-Leoneti, V.; Campo, V. L.; Gomes, A. S.; Field, R. A.; Carvalho, I.; Tetrahedron 2010, 66, 9475.

15. Rodionov, V. O.; Fokin, V. V.; Finn, M. G.; Angew. Chem., Int. Ed. 2005, 44, 2210.

16. Bock, V. D.; Hiemstra, H.; Maarseveen, J. H.; Eur. J. Org. Chem. 2006, 51.

17. Sun, S.; Wu, P.; J. Phys. Chem. A 2010, 114, 8331.

18. Binder, W. H.; Sachsenhofer, R.; Macromol. Rapid Commun. 2008, 29 , 952.

19. Zhang, L.; Chen, X.; Xue, P.; Sun, H. H. Y.; Williams, I. D.; Sharpless, K. B.; Fokin, V. V.; Jia, G.; J. Am. Chem. Soc., 2005, 127, 15998.

20. Sreedhar, B.; Reddy, P. S.; Synth. Commun. 2007, 37, 805.

21. Bock, V. D.; Perciaccante, R.; Jansen, T. P.; Hiemstra, H.; van Maarseveen, J. H.; Org. Lett. 2006, 8, 919.

22. Punna, S.; Kaltgrad, E.; Finn, M. G.; Bioconjugate Chem. 2005, 16, 1536.

23. Wróblewski, A. E.; Glowacka, I. E.; Tetrahedron: Asymmetry 2005, 16, 4056.

24. Kuang, G. C.; Michaels, H. A.; Simmons, J. T.; Clark, R. J.; Zhu, L.; J. Org. Chem. 2010, 75, 6540 .

25. Trabocchi, A.; Menchi, G.; Cini, N.; Bianchini, F.; Raspanti, S.; Bottoncetti, A.; Pupi, A.; Calorini, L.; Guarna, A.; J. Med. Chem. 2010, 53,7119 .

26. Aucagne, V.; Hänni, K. D.; Leigh, D. A.; Lusby, P. J.; Walker, D. B.; J. Am. Chem. Soc. 2006, 128, 2186.

27. Gupta, S. S.; Kuzelka, J.; Singh, P.; Lewis, W. G.; Manchester, M.; Finn, M. G.; Bioconjugate Chem. 2005, 16, 1572.

28. Hein, J. E.; Tripp, J. C.; Krasnova, L. B.; Sharpless, K. B.; Fokin, V. V.; Angew. Chem., Int. Ed. 2009, 48, 8018.

29. Appukkuttan, P.; Dehaen, W.; Fokin, V. V.; van der Eycken, E.; Org. Lett. 2004, 6, 4223

30. Pachón, L. D.; van Maarseveen, J. H.; Rothenberg, G.; Adv. Synth. Catal. 2005, 347, 811

31. Wan, Q.; Chen, J.; Chen, G.; Danishefsky, S. J.; J. Org. Chem. 2006, 71, 8244 .

32. Yoon, K.; Goyal, P.; Weck M.; Org. Lett. 2007, 9, 2051.

33. Guezguez, R.; Bougrin, K.; El Akri, K.; Benhida, R.; Tetrahedron Lett. 2006, $47,4807$.

34. Cravotto, G.; Fokin, V. V.; Garella, D.; Binello, A.; Boffa, L; Barge, A.; J. Comb. Chem. 2010, 12, 13.

35. Cintas, P.; Barge, A.; Tagliapietra, S.; Boffa, L.; Cravotto, G.; Nature 2010, 5, 607 .

36. Balderas, F. P.; Muñoz, M. O.; Sanfrutos, J. M.; Mateo, F. H.; Flores, F. G. C.; Asín, J. A. C.; García, J. I.; González, F. S.; Org. Lett. 2003, 5, 1951.

37. Wu, P.; Feldman, A. K.; Nugent, A. K.; Hawker, C. J.; Scheel, A.; Voit, B.; Pyun, J.; Fréchet, J. M. J.; Sharpless, K. B.; Fokin, V. V.; Angew. Chem., Int. Ed. 2004, 43, 3928.

38. Wu, P.; Malkoch, M.; Hunt, J. N.; Vestberg, R.; Kaltgrad, E.; Finn, M. G.; Fokin, V. V.; Sharpless, K. B.; Hawker, C. J.; Chem. Commun. 2005, 5775 .

39. Gonda, Z.; Novák, Z.; Dalton Trans. 2010, 39, 726.

40. Díez-González, S.; Nolan, S. P.; Angew. Chem., Int. Ed. 2008, 120, 9013.

41. Chan, T. R.; Hilgraf, R.; Sharpless, K. B.; Fokin, V. V.; Org. Lett. 2004, 6, 2853.

42. Amo, D. S.; Wang, W.; Jiang, H.; Besanceney, C.; Yan, A. C.; Levy, M.; Liu, Y.; Marlow, F. L.; Wu, P.; J. Am. Chem. Soc. 2010, 132, 16893.

43. Kamijo, S.; Jin, T.; Yamamoto, Y.; Tetrahedron Lett. 2004, 45, 689

44. Isobe, H.; Cho, K.; Solin, N.; Werz, D. B.; Seeberger, P. H.; Nakamura, E.; Org. Lett. 2007, 9, 4611.

45. Pereira, G. R.; Santos, L. J.; Luduvico, I.; Alves, R. B.; Freitas, R. P.; Tetrahedron Lett. 2010, 51, 1022.
46. Kuang, G. C.; Michaels, H. A.; Simmons, J. T.; Clark, R. J.; Zhu, L.; J. Org. Chem. 2010, 75, 6540.

47. Hasegawa, T.; Umeda, M.; Numata, M.; Li, C.; Bae, A. H.; Fujisawa,T.; Haraguchi, S.; Sakurai, K.; Shinkai, S.; Carbohydr. Res. 2006, 341, 35.

48. Molteni, G.; Bianchi, C. L.; Marinoni, G.; Santo, N.; Ponti, A.; New J. Chem. 2006, 30, 1137.

49. Gupta, S. S.; Raja, K. S.; Kaltgrad, E.; Strable, E.; Finn, M. G.; Chem. Commun. 2005, 4315.

50. Lipshutz, B. H.; Taft, B. R.; Angew. Chem. Int. Ed. 2006, 118, 8415.

51. Tron, G. C.; Pirali, T.; Billington, R. A.; Canonico, P. L.; Sorba, G.; Genazzani, A. A.; Medicinal Research Reviews 2008, 28, 278.

52. Agut, W.; Taton, D.; Lecommandoux, S.; Macromolecules 2007, 40, 5653; Agut, W.; Agnaou, R.; Lecommandoux, S.; Taton, D.; Macromol. Rapid Commun. 2008, 29, 1147.

53. van Camp, W.; Germonpré, V.; Mespouille, L.; Dubois, P.; Goethals, E. J.; Du Prez, F. E.; React. Funct. Polym. 2007, 67, 1168.

54. He, X.; Liang, L.; Xie, M.; Zhang, Y.; Lin, S.; Yan, D.; Macromol. Chem. Phys. 2007, 208, 1797.

55. Durmaz, H.; Dag, A.; Altintas, O.; Erdogan, T.; Hizal, G.; Tunca, U.; Macromolecules 2007, 40, 191.

56. Dag, A.; Durmaz, H.; Hizal, G.; Tunca, U.; J. Polym. Sci., Part A: Polym. Chem. 2008, 46, 302

57. Lee, B. Y.; Park, S. R.; Jeon, H. B.; Kim, K. S.; Tetrahedron Lett. 2006 , 47, 5105 .

58. Binder, W. H.; Sachsenhofer, R.; Macromol. Rapid Commun. 2007, 28, 15.

59. Fleming, D. A.; Thode, C. J.; Williams, M. E.; Chem. Mater. 2006, 18 2327

60. Bew, S. P.; Brimage, R. A.; L'Hermite, N.; Sharma, S. V.; Org. Lett. 2007, 9, 3713.

61. Dedola, S.; Nepogodiev, S. A.; Field, R. A.; Org. Biomol. Chem. 2007, 5,1006 .

62. Turner, R. A.; Oliver, A. G.; Lokey, R. S.; Org. Lett. 2007, 9, 5011.

63. Golas, P. L.; Tsarevsky, N. V.; Matyjaszewski, K.; Macromol. Rapid. Commun. 2008, 29, 1167.

64. Molander, G. A.; Ham, J.; Org. Lett. 2006, 8, 2767.

65. Barbosa, F. C. G.; Oliveira, R. N.; J. Braz. Chem. Soc. 2011, 22, 592.

66. Feldman, A. K.; Colasson, B.; Sharpless, K. B.; Fokin, V. V.; J. Am. Chem. Soc. 2005, 127, 13444.

67. Oladeinde, O. A.; Hong, S. Y.; Holland, R. J.; Maciag, A. E.; Keefer, L. K.; Saavedra, J. E.; Nandurdikar, R. S.; Org. Lett. 2010, 12, 4256.

68. Angell, Y.; Burgess, K.; Angew. Chem., Int. Ed. 2007, 46, 3649.

69. Gerard, B.; Ryan, J.; Beeler, A. B.; Porco, Jr., J. A.; Tetrahedron 2006 , 62, 6405.

70. Pérez-Castro, I.; Caamaño, O.; Fernández, F.; García, M. D.; López, C.; De Clercq, E.; Org. Biomol. Chem. 2007, 5, 3805.

71. Li, L.; Zhang, G.; Zhu, A.; Zhang, L.; J. Org. Chem. 2008, 73, 3630.

72. Ackermann, L.; Potukuchi, H. K.; Org. Biomol. Chem. 2010, 8, 4503.

73. Wu, Y. M.; Deng, J.; Chen, Q. Y.; Synlett 2006, 4, 645; Wu, Y. M.; Deng, J.; Li, Y.; Chen, Q. Y.; Synthesis 2005, 8, 1314.

74. Mindt, T. L.; Shibli, R.; J. Org. Chem. 2007, 72, 10247.

75. Cassidy, M. P.; Raushel, J.; Fokin, V. V.; Angew. Chem., Int. Ed. 2006, 45, 3154 .

76. Bertrand, P.; Gesson, J. P.; J. Org. Chem. 2007, 72, 3596.

77. Zhang, X.; Hsung, R. P.; You, L.; Org. Biomol. Chem. 2006, 4, 2679.

78. Zhang, X.; Li, H.; You, L.; Tang, Y.; Hsung, R. P.; Adv. Synth. Catal. 2006, 348, 2437.

79. IJsselstijn, M.; Cintrat, J. C.; Tetrahedron 2006, 62, 3837.

80. Whiting, M.; Tripp, J. C.; Lin, Y. C.; Lindstrom, W.; Olson, A. J.; Elder, J. H.; Sharpless, K. B.; Fokin, V. V.; J. Med. Chem. 2006, 49, 7697.

81. Appendino, G.; Bacchiega, S.; Minassi, A.; Cascio, M. G.; Petrocellis, L.; Marzo, V.; Angew. Chem., Int. Ed. 2007, 46, 9312.

82. Pagliali, F.; Pirali, T.; Del Grosso, E.; Brisco, R. D.; Tron, G. C.; Sorba, G.; Genazzani, A. A.; J. Med. Chem. 2006, 49, 467. 
83. Silva Jr., E. N.; Barreto, R. F. S. M.; Pinto, M. C. F. R.; Silva, R. S. F.; Teixeira, D. V.; Souza, M. C. B. V.; Simone, C. A.; De Castro S. L.; Ferreira, V. F.; Pinto, A. V.; Eur. J. Med. Chem. 2008, 43, 1774.

84. Kamal, A.; Shankaraiah, N.; Devaiah, V.; Reddy, K. L.; Juvekar, A.; Sen, S.; Kurian, N.; Zingde, S.; Bioorg. Med. Chem. Lett. 2008, 18, 1468.

85. Colombano, G.; Travelli, C.; Galli, U.; Caldarelli, A.; Chini, M. G.; Canônico, P. L.; Sorba, G.; Bifulco, G.; Tron, G. C.; Genazzani, A. A.; J. Med. Chem. 2010, 53, 616.

86. Bakunov, S. A.; Bakunova, S. M.; Wenzler, T.; Ghebru, M.; Werbovetz, K. A.; Brun, R.; Tidwell, R. R.; J. Med. Chem. 2010, 53, 254.

87. Carvalho, I.; Andrade, P.; Campo, V. L.; Guedes, P. M. M.; Costa, R. S.; Silva, J. S.; Schenkman, S.; Dedola, S.; Hill, L.; Rejzek, M.; Nepogodiev, S. A.; Field, R. A.; Bioorg. Med. Chem. 2010, 18, 2412.

88. Silva Jr., E. N.; Moura, M. A. B. F.; Pinto, A. V.; Pinto, M. C. F. R.; Souza, M. C. B. V.; Araújo, A. J.; Pessoa, C.; Lotufo, L. V. C.; Montenegro, R. C.; Moraes, M. O.; Ferreira, V. F.; Goulart, O. F.; J. Braz. Chem. Soc. 2009, 20, 635.

89. Gallardo, H.; Conte, G.; Bryk, F.; Lourenço, M. C. S.; Costa, M. S.; Ferreira, V. F.; J. Braz. Chem. Soc. 2007, 18, 1285.

90. Pore, V. S.; Aher, N. G.; Kumar, M.; Shukla, P. K.; Tetrahedron 2006, 62,11178 .

91. Guantai, E. M.; Ncokazi, K.; Egan, T. J.; Gut, J.; Rosenthal, P. J.; Smith, P. J.; Chibale, K.; Bioorg. Med. Chem. 2010, 18, 8243.

92. Song, Z.; He, X. P.; Jin, X. P.; Gao, L. X.; Sheng, L.; Zhou, Y. B.; Li, J.; Chen, G. R.; Tetrahedron Lett. 2011, 52, 894.

93. Díaz, L.; Bujons, J.; Casas, J.; Llebaria, A.; Delgado, A.; J. Med. Chem. 2010, 53, 5248 .

94. Mohan, S.; McAtamney, S.; Haselhorst, T.; Itzstein, M.; Pinto, B. M.; J. Med. Chem. 2010, 53, 7377.

95. Galli, U.; Ercolano, E.; Carraro, L.; Roman, C. R. B.; Sorba, G.; Canônico, P. L.; Genazzani, A. A.; Tron, G. C.; Billington, R. A.; ChemMedChem 2008, 3, 771.

96. Santos, F. C.; Cunha, A. C.; Souza, M. C. B. V.; Tomé, A. C.; Neves, M. G. P. M. S.; Ferreira, V. F.; Cavaleiro, J. A. S.; Tetrahedron Lett. 2008, 49, 7268.

97. Wang, Q.; Chan, T. R.; Hilgraf, R.; Fokin, V. V.; Sharpless, K. B.; Finn, M. G.; J. Am. Chem. Soc. 2003, 125, 3192.

98. Deiters, A.; Cropp, T. A.; Mukherji, M.; Chin, J. W.; Anderson, J. C.; Schultz, P. G.; J. Am. Chem. Soc. 2003, 125, 11782

99. Dirks, A. J. T.; van Berkel, S. S.; Hatzakis, N. S.; Opsteen, J. A.; van Delft, F. L.; Cornelissen, J. J. L. M.; Rowan, A. E.; van Hest, J. C. M.; Rutjes, F. P. J. T.; Nolte, R. J. M.; Chem. Commun. 2005, 33, 4172.

100. Kosiova, I.; Kovackova, S.; Kois, P.; Tetrahedron 2007, 63, 312.

101. Marik, J.; Sutcliffe, J. L.; Tetrahedron. Lett. 2006, 47, 6681.

102. Mamat, C.; Ramenda, T.; Wuest, F. R.; Mini-Rev. Org. Chem. 2009, 6, 21.

103. Sudhir, V. S.; Venkateswarlu, C.; Musthafa, O. T. M.; Sampath, S.; Chandrasekaran, S.; Eur. J. Org. Chem. 2009, 13, 2120.

104. Yang, J.; Ye, T.; Ma, D.; Zhang, Q.; Synth. Met. 2011, 161, 330.

105. Ornelas, C.; Aranzaes, J. R.; Cloutet, E.; Alves, S.; Astruc, D.; Angew. Chem., Int. Ed. 2007, 46, 872.

106. Camponovo, J.; Ruiz, J.; Cloutet, E.; Astruc, D.; Chem. Eur. J. 2009, 15, 2990.

107. Yim, C. B.; Boerman, O. C.; Visser, M.; Jong, M.; Dechesne, A. C.; Rijkers, D. T. S.; Liskamp, R. M. J.; Bioconjugate Chem. 2009, 20, 1323.

108. Zhu, Y.; Huang, Y.; Meng, W. D.; Li, H.; Qing, F. L.; Polymer 2006, 47, 6272.

109. Deng, G.; Ma, D.; Xu, Z.; Eur. Polym. J. 2007, 43, 1179.

110. Whittaker, M. R.; Urbani, C. N.; Monteiro, M. J.; J. Am. Chem. Soc. 2006, 128, 11360.

111. Chakraborty, S.; Keightley, A.; Dusevich, V.; Wang, Y.; Peng, Z.; Chem. Mater. 2010, 22, 3995
112. Díaz, D. D.; Punna, S.; Holzer, P.; McPherson, A. K.; Sharpless, K. B.; Fokin, V. V.; Finn, M. G.; J. Polym. Sci., Part A: Polym. Chem. 2004, 42, 4392.

113. Liu, Y.; Díaz, D. D.; Accurso, A. A.; Sharpless, K .B.; Fokin, V. V.; Finn, M. G.; J. Polym. Sci., Part A: Polym. Chem. 2007, 45, 5182.

114. Luo, L.; Frisbie, C. D.; J. Am. Chem. Soc. 2010, 132, 8854.

115. Leal, M. P.; Assali, M.; Fernández, I.; Khiar, N.; Chem. Eur. J. 2011, 17, 1828.

116. González-Vera, J. A.; Lukovic, E.; Imperiali, B.; J. Org. Chem. 2009, 74, 7309.

117. Michel, K.; Büther, K.; Law, M. P.; Wagner, S.; Schober, O.; Hermann, S.; Schäfers, M.; Riemann, B.; Höltke, C.; Kopka, K.; J. Med. Chem. 2011, 54, 939.

118. van Steenis, D. J. V. C.; David, O. R. P.; van Strijdonck, G. P. F.; van Maarseveen, J. H.; Reek, J. N. H.; Chem. Commun. 2005, 34, 4333.

119. Guo, Z.; Lei, A.; Liang, X.; Xu, Q.; Chem. Commun. 2006, 43, 4512.

120. Slater, M.; Snauko, M.; Svec, F.; Fréchet, J. M. J.; Anal. Chem. 2006, $78,4969$.

121. Zhang, Y.; Guo, Z.; Ye, J.; Xu, Q.; Liang, X.; Lei, A.; J. Chromatogr., A 2008, 1191, 188.

122. Fu, Q.; Guo, Z.; Liang, T.; Zhang, X.; Xu, Q.; Liang, X.; Anal. Methods 2010, 2, 217.

123. Park, S. M.; Lee, Y. S.; Kim, B. H.; Chem. Commun. 2003, 23, 2912.

124. Malkoch, M.; Vestberg, R.; Gupta, N.; Mespouille, L.; Dubois, P.; Mason, A. F.; Hedrik, J. L.; Liao, Q.; Frank, C. W.; Kingsbury, K.; Hawker, C. J.; Chem. Commun. 2006, 2774.

125. Díaz, D. D.; Rajagopal, K.; Strable, E.; Schneider, J.; Finn, M. G.; J. Am. Chem. Soc. 2006, 128, 6056.

126. Liu, J. Y.; El-Khouly, M. E.; Fukuzumi, S.; Ng, D. K. P.; Chem. Eur. J. 2011, 17, 1605 .

127. Palacin, T.; Khanh, H. L.; Jousselme, B.; Jegou, P.; Filoramo, A.; Ehli, C.; Guldi, D. M.; Campidelli, S.; J. Am. Chem. Soc. 2009, 131, 15394.

128. Hizume, Y.; Tashiro, K.; Charvet, R.; Yamamoto, Y.; Saeki, A.; Seki, S.; Aida, T.; J. Am. Chem. Soc. 2010, 132, 6628.

129. Freitas, R. P.; Iehl, J.; Delavaux-Nicot, B.; Nierengarten, J. F.; Tetrahedron 2008, 64, 11409.

130. Ryu, E. H.; Zhao, Y.; Org. Lett. 2005, 7, 1035.

131. Bew, S. P.; Brimage, R. A.; L'Hermite, N.; Sharma, S. V.; Org. Lett. 2007, 9, 3713 .

132. Li, H.; Zhan J.; Chen, M.; Tian, D.; Zou, Z.; J. Incl. Phenom. Macrocycl. Chem. 2010, 66, 43 .

133. Ni, X. L.; Wang, S.; Zeng, X.; Tao, Z.; Yamato, T.; Org. Lett. 2011, 13, 552.

134. Roice, M.; Johannsen, I.; Meldal, M.; QSAR Comb. Sci. 2004, 23, 662.

135. Bodine, K. D.; Gin, D. Y.; Gin, M. S.; J. Am. Chem. Soc. 2004, 126, 1638.

136. Bodine, K. D.; Gin, D. Y.; Gin, M. S.; Org. Lett. 2005, 7, 4479.

137. Aucagne, V.; Berná, J.; Crowley, J. D.; Goldup, S. M.; Hänni, K. D.; Leigh, D. A.; Lusby, P. J.; Ronaldson, V. E.; Slawin, A. M. Z.; Viterisi, A.; Walker, D. B.; J. Am. Chem. Soc. 2007, 129, 11950.

138. Aucagne, V.; Hänni, K. D.; Leigh, D. A.; Lusby, P. J.; Walker, D. B.; J. Am. Chem. Soc. 2006, 128, 2186.

139. Collin, J. P.; Durola, F.; Frey, J.; Heitz, V.; Reviriego, F.; Sauvage, J. P.; Trolez, Y.; Rissanen, K.; J. Am. Chem. Soc. 2010, 132, 6840.

140. Megiatto Jr., J. D.; Schuster, D. I.; J. Am. Chem. Soc. 2008, 130, 12872.

141. Goldup, S. M.; Leigh, D. A.; Long, T.; McGonigal, P. R.; Symes, M. D.; Wu, J.; J. Am. Chem. Soc. 2009, 131, 15924.

142. Megiatto Jr., J. D.; Schuster, D. I.; Abwander, S.; Miguel, G.; Guldi, D. M.; J. Am. Chem. Soc. 2010, 132, 3847.

143. Morales-Sanfrutos, J.; Ortega-Muñoz, M.; Lopes-Jaramillo, J.; Hernandez-Mateo, F.; Santoyo-Gonzales, F.; J. Org. Chem. 2008, 73, 7772 . 
144. Niu, Y. N.; Yan, Z. Y.; Li, G. Q.; Wei, H. L.; Gao, G. L.; Wu, L. Y.; Liang, Y. M.; Tetrahedron: Assymetry 2008, 19, 912.

145. Diallo, A. K.; Ornelas, C.; Salmon, L.; Aranzaes, J. R.; Astruc, D.; Angew. Chem., Int. Ed. 2007, 46, 8644.

146. Detz, R. J.; Heras, S. A.; Gelder, R.; van Leeuwen, P. W. N. M.; Hiemstra, H.; Reek, J. N. H.; van Maarseveen, J. H.; Org. Lett. 2006, 8, 3227.

147. Gissibl, A.; Padié, C.; Hager, M.; Jaroschik, F.; Rasappan, R.; Yañez, E. C.; Turrin, C. O.; Caminade, A. M.; Majoral, J. P.; Reiser, O.; Org. Lett. 2007, 9, 2895.

148. Zhang, Q.; Takacs, J. M.; Org. Lett. 2008, 10, 545.

149. Manetsch, R.; Krasinski, A.; Radic, Z.; Raushel, J.; Taylor, P.; Sharpless, K. B.; Kolb, H. C.; J. Am. Chem. Soc. 2004, 126, 12809.

150. Gil, M. V.; Arévalo, M. J.; López, O.; Synthesis 2007, 11, 1589.

151. Holub, J. M.; Kirshenbaum, K.; Chem. Soc. Rev. 2010, 39, 1325.

152. Cho, S.; Oh, S.; Um, Y.; Jung, J. H.; Ham, J.; Shin, W. S.; Lee, S.; Bioorg. Med. Chem. 2009, 19, 382.

153. Vasilevsky, S. F.; Govdi, A. I.; Sorokina, I. V.; Tolstikova, T. G.; Baev, D. S.; Tolstikov, G. A.; Mamatuyk, V. I.; Alabugin, I. V.; Bioorg. Med. Chem. 2011, 21, 62.

154. Ferreira, S. B.; Sodero, A. C. R.; Cardoso, M. F. C.; Lima, E. S.; Kaiser, C. R.; Jr., F. P. S.; J. Med. Chem. 2010, 53, 2364

155. Brik, A.; Muldoon, J.; Lin, Y. C.; Elder, J. H.; Goodsell, D.; Olson, A. J.; Fokin, V. V.; Sharpless, K. B.; Wong, C. H.; ChemBioChem 2003, 4, 1246.
156. Brik, A.; Alexandratos, J.; Lin, Y. C.; Elder, J. H.; Olson, A. J.; Wlodawer, A.; Goodsell, D. S.; Wong, C. H.; ChemBioChem 2005, 6, 1167.

157. Tornфe, C. W.; Sanderson, S. J.; Mottram, J. C.; Coombs, G. H.; Meldal, M.; J. Comb. Chem. 2004, 6, 312.

158. Srinivasachari, S.; Liu, Y.; Zhang, G.; Prevette, L.; Reineke, T. M.; $J$. Am. Chem. Soc. 2006, 128, 8176.

159. Xie, J.; Seto, C. T.; Bioorg. Med. Chem. 2007, 15, 458.

160. Lahann, J.; Click chemistry for biotechnology and materials science, John Wiley \& Sons Ltd: Chichester, 2009.

161. Kalia, J.; Raines, R. T.; Curr. Org. Chem. 2010, 14, 138.

162. Wang, T.; Guo, Z.; Curr. Med. Chem. 2006, 13, 525.

163. Moses, J. E.; Moorhouse, A. D.; Chem. Soc. Rev. 2007, 36, 1249.

164. Frank, G.; Kakkar, A. K.; Chem. Soc. Rev. 2010, 39, 1536.

165. Shirota, Y.; J. Mater. Chem. 2000, 10, 1.

166. Friend, R. H.; Gymer, R. W.; Holmes, A. B.; Burroughes, J. H.; Marks, R. N.; Taliani, C.; Bradley, D. D. C.; Dos Santos, D. A.; Brédas, J. L.; Logdlund, M.; Salaneck, W. R.; Nature 1999, 397, 121.

167. Yang, Y.; Turnbull, G. A.; Samuel, I. D. W.; Appl. Phys. Lett. 2008, 92, 163306.

168. Jiang, Y.; Okamoto, T.; Becerril, H. A.; Hong, S.; Tang, M. L.; Mayer, A. C.; Parmer, J. A.; McGehee, M. D.; Bao, Z.; Macromolecules 2010, 43,6361 .

169. S'Heeren, G.; Derhaeg, L.; Verbiest, T.; Samyn, C.; Persoons, A.; Macromol. Symp. 1993, 69, 193.

170. Lutz, J. F.; Zarafshani, Z.; Adv. Drug Delivery Rev. 2008, 60, 958. 